\title{
CLASSIFICATION OF A SUBCLASS OF QUASILINEAR TWO-DIMENSIONAL LATTICES BY MEANS OF CHARACTERISTIC ALGEBRAS
}

\author{
M.N. KUZNETSOVA
}

\begin{abstract}
We consider a classification problem of integrable cases of the Toda type twodimensional lattices $u_{n, x y}=f\left(u_{n+1}, u_{n}, u_{n-1}, u_{n, x}, u_{n, y}\right)$. The function $f=f\left(x_{1}, x_{2}, \cdots x_{5}\right)$ is assumed to be analytic in a domain $D \subset \mathbb{C}^{5}$. The sought function $u_{n}=u_{n}(x, y)$ depends on real $x, y$ and integer $n$. Equations with three independent variables are complicated objects for study and especially for classification. It is commonly accepted that for a given equation, the existence of a large class of integrable reductions indicates integrability. Our classification algorithm is based on this observation. We say that a constraint $u_{0}=\varphi(x, y)$ defines a degenerate cutting off condition for the lattice if it divides this lattice into two independent semi-infinite lattices defined on the intervals $-\infty<n<0$ and $0<n<+\infty$, respectively. We call a lattice integrable if there exist cutting off boundary conditions allowing us to reduce the lattice to an infinite number of hyperbolic type systems integrable in the sense of Darboux. Namely, we require that lattice is reduced to a finite system of such kind by imposing degenerate cutting off conditions at two different points $n=N_{1}$, $n=N_{2}$ for arbitrary pair of integers $N_{1}, N_{2}$. Recall that a system of hyperbolic equations is called Darboux integrable if it admits a complete set of integrals in both characteristic directions. An effective criterion of the Darboux integrability of the system is connected with properties of an associated algebraic structures. More precisely, the characteristic LieRinehart algebras assigned to both characteristic directions have to be of a finite dimension. Since the obtained hyperbolic system is of a very specific form, the characteristic algebras are effectively studied. Here we focus on a subclass of quasilinear lattices of the form

$$
u_{n, x y}=p\left(u_{n-1}, u_{n}, u_{n+1}\right) u_{n, x}+r\left(u_{n-1}, u_{n}, u_{n+1}\right) u_{n, y}+q\left(u_{n-1}, u_{n}, u_{n+1}\right) .
$$
\end{abstract}

Keywords: two-dimensional lattice, integrable reduction, characteristic Lie algebra, degenerate cutting off condition, Darboux integrable system, $x$-integral.

Mathematics Subject Classification: 37K10, 37K30, 37D99

\section{INTRODUCTION}

The problem of classifying integrable two-dimensional chains of the form

$$
u_{n, x y}=f\left(u_{n+1}, u_{n}, u_{n-1}, u_{n, x}, u_{n, y}\right), \quad-\infty<n<\infty,
$$

is topical and currently remains open. The function $f=f\left(x_{1}, x_{2}, \cdots x_{5}\right)$ is assumed to be analytic in a domain $D \subset \mathbb{C}^{5}$, and the sought function $u_{n}=u_{n}(x, y)$ depends on real $x, y$ and integer $n$.

In this paper we focus on the following subclass of quasilinear lattices (1.1):

$$
u_{n, x y}=p\left(u_{n+1}, u_{n}, u_{n-1}\right) u_{n, x}+r\left(u_{n+1}, u_{n}, u_{n-1}\right) u_{n, y}+q\left(u_{n+1}, u_{n}, u_{n-1}\right), \quad-\infty<n<\infty \text {. }
$$

M.N. Kuznetsova, Classification of a Subclass of Quasilinear Two-Dimensional Lattices by means of Characteristic Algebras.

(C) KuZnetsova M.N. 2019.

Submitted April 3, 2019. 
Here functions $p\left(x_{1}, x_{2}, x_{3}\right), r\left(x_{1}, x_{2}, x_{3}\right), q\left(x_{1}, x_{2}, x_{3}\right)$ are assumed to be analytic in a domain $D \subset \mathbb{C}^{3}$.

Equations with three independent variables are complicated objects for study and especially for classification. Currently, there are different approaches to studying integrable multidimensional equations [1]-10]. The presence of a wide class of integrable reductions indicates the integrability of the equation. This fact is often used in the study of multidimensional equations, see [1, 2, 3], where the existence of integrable reductions of a hydrodynamic type is taken to determine the integrability. Here we use a similar idea by treating integrability as the presence of an infinite sequence of Darboux integrable hyperbolic systems.

In describing Darboux integrable systems of hyperbolic equations of a special type, the concept of the characteristic Lie algebra [11, 12, 13] was used a lot. The transition to a more general characteristic Lie-Rinehart algebra opens up new possibilities [14]-[18].

The characteristic Lie algebra for two-dimensional lattices was introduced in [19]. Namely, the structure of this algebra was described for two-dimensional Toda lattice. It was observed in paper [16] that any integrable lattice of the form (1.1) admits a so-called degenerate cutting off boundary conditions. When such kind boundary conditions are imposed at two different points $n=N_{1}$ and $n=N_{2}$, the lattice reduces to a Darboux integrable system of the hyperbolic type equations. In our works [16], [17], [18], we suggested and developed a classification algorithm based on this observation. Let us briefly discuss the essence of the method.

We say that a constraint

$$
u_{0}=\varphi(x, y)
$$

defines a degenerate cutting off condition for lattice $(1.1)$ if it divides 1.1 into two independent semi-infinite lattices defined on the intervals $-\infty<n<0$ and $0<n<+\infty$, respectively.

Definition 1.1. Lattice (1.1) is called integrable if there exist functions $\varphi_{1}$ and $\varphi_{2}$ such that for any pair of integers $N_{1}, N_{2}$, where $N_{1}<N_{2}-1$, the hyperbolic system

$$
\begin{aligned}
& u_{N_{1}}=\varphi_{1}(x, y), \\
& u_{n, x y}=f\left(u_{n+1}, u_{n}, u_{n-1}, u_{n, x}, u_{n, y}\right), \quad N_{1}<n<N_{2}, \\
& u_{N_{2}}=\varphi_{2}(x, y)
\end{aligned}
$$

obtained from lattice (1.1) by imposing degenerate boundary conditions is integrable in the sense of Darboux.

Recall that a system of hyperbolic equations is called Darboux integrable if it admits a complete set of integrals in both characteristic directions, see [14], [15]. An effective criterion of the Darboux integrability of the system is connected with properties of an associated algebraic structures. More precisely, the characteristic Lie-Rinehart algebras [20, 21] assigned to both characteristic directions have to be of a finite dimension. Since the obtained hyperbolic system is of a very specific form, this allows us to study effectively the characteristic algebras. The method was shown to be effective in our articles [17], [18]. A large class of the integrable lattices of form (1.1) was represented in [22], where they were studied in the framework of the symmetry approach. It is remarkable that all equations of this class turned out to be integrable in the sense of Definition 1.1. Another argument in favor of our definition is that the resulting hyperbolic systems admit explicit solutions, which are extended to solutions of the original nonlinear chain. So, the chains integrable in our sense have a very wide class of explicit solutions.

In this article we continue the study initiated in [17, [18, where the integrable in the sense of Definition 1.1 cases of the two-dimensional quasilinear lattices of the form

$$
u_{n, x y}=\alpha_{n} u_{n, x} u_{n, y}+p_{n} u_{n, x}+r_{n} u_{n, y}+q_{n},
$$


were described under the non-degeneracy condition $\frac{\partial \alpha_{n}}{\partial u_{n \pm 1}} \neq 0$. Here the coefficients depend on three successive variables

$$
\begin{aligned}
& \alpha_{n}=\alpha\left(u_{n+1}, u_{n}, u_{n-1}\right), \quad p_{n}=p\left(u_{n+1}, u_{n}, u_{n-1}\right), \\
& r_{n}=r\left(u_{n+1}, u_{n}, u_{n-1}\right), \quad q_{n}=q\left(u_{n+1}, u_{n}, u_{n-1}\right) .
\end{aligned}
$$

We mention review [23], where a complete classification of lattices of the form

$$
u_{n, x x}=f\left(u_{n-1}, u_{n}, u_{n+1}, u_{n, x}\right), \quad \frac{\partial f}{\partial u_{n+1}} \frac{\partial f}{\partial u_{n+1}} \neq 0,
$$

was presented. In our paper [18], we found two new equations of form (1.3), which were integrable in the sense of Definition 1.1. We note that these equations were two-dimensional generalizations of the equations from the list in paper [23].

Now we focus on a particular case (1.2) of lattice (1.3), as $\alpha_{n}$ vanishes identically. We suppose that the following conditions are satisfied: at least one of the following derivatives is non-zero:

$$
\begin{aligned}
\frac{\partial r_{n}}{\partial u_{n+1}} & \neq 0 & \text { or } & \frac{\partial r_{n}}{\partial u_{n-1}} & \neq 0, \\
\frac{\partial p_{n}}{\partial u_{n+1}} & \neq 0 & \text { or } & \frac{\partial p_{n}}{\partial u_{n-1}} & \neq 0 .
\end{aligned}
$$

The main result of this paper is as follows.

Theorem 1.1. If chain (1.2), (1.4) is integrable in the sense of Definition 1.1, then by point transformations it is reduced to one of the following forms:

$$
\begin{aligned}
& u_{n, x y}=\left(e^{u_{n}-u_{n-1}}-e^{u_{n+1}-u_{n}}\right) u_{n, y}, \\
& u_{n, x y}=\left(-u_{n+1}+2 u_{n}-u_{n-1}\right) u_{n, y} .
\end{aligned}
$$

Lattices (1.6), (1.7) were known before [22]. Condition (1.5) implies that lattices obtained under classification procedure coincide with these lattices up to the change $x \leftrightarrow y$.

In the next section we describe briefly a theoretical base of the main research method; a detailed explanation was presented in [17, 18].

\section{PRELIMINARIES}

According Definition 1.1, there exist cutting off conditions at two points that reduce 1.2 to the finite hyperbolic type system:

$$
\begin{aligned}
& u_{-1}=\varphi_{1}, \\
& u_{n, x y}=p_{n} u_{n, x}+r_{n} u_{n, y}+q_{n}, \quad 0 \leqslant n \leqslant N, \\
& u_{N+1}=\varphi_{2} .
\end{aligned}
$$

Here $p_{n}=p\left(u_{n-1}, u_{n}, u_{n+1}\right), r_{n}=r\left(u_{n-1}, u_{n}, u_{n+1}\right), q_{n}=q\left(u_{n-1}, u_{n}, u_{n+1}\right)$.

We recall that a hyperbolic system of partial differential equations (2.1) is integrable in the sense of Darboux if it admits a complete set of functionally independent $x$ - and $y$-integrals (see [14]). A function $I$ depending on finitely many dynamical variables $\mathbf{u}, \mathbf{u}_{x}, \mathbf{u}_{x x}, \ldots$ is called $y$ integral if it solves the equation $D_{y} I=0$ (see [14]), where $D_{y}$ is the operator of total derivative with respect to variable $y$ and $\mathbf{u}$ is a vector with coordinates $u_{0}, u_{1}, \ldots, u_{N}$. Since system (2.1) is autonomous, we consider autonomous $y$-integrals depending at least on one of the dynamical variables $\mathbf{u}, \mathbf{u}_{x}, \mathbf{u}_{x x}, \ldots$

We suppose that system (2.1) is Darboux integrable and denote by $I\left(\mathbf{u}, \mathbf{u}_{x}, \ldots\right)$ its nontrivial $y$-integral. By definition, $I$ solves the equation $D_{y} I=0$. Let us calculate an action of the 
operator $D_{y}$ on functions of the form $I\left(\mathbf{u}, \mathbf{u}_{x}, \ldots\right)$. It is determined by the rule $D_{y} I=Y I$, where

$$
Y=\sum_{i=0}^{N}\left(u_{i, y} \frac{\partial}{\partial u_{i}}+f_{i} \frac{\partial}{\partial u_{i, x}}+f_{i, x} \frac{\partial}{\partial u_{i, x x}}+\cdots\right) .
$$

Here $f_{i}=p_{i} u_{i, x}+r_{i} u_{i, y}+q_{i}$ is the right hand side of lattice (1.2). Therefore, the function $I$ satisfies the equation $Y I=0$. Coefficients of the equation $Y I=0$ depend on the variables $u_{i, y}$, whereas a solution $I$ is independent of $u_{i, y}$. Hence, $I$ satisfies the system of linear equations:

$$
Y I=0, \quad X_{j} I=0, \quad j=0, \ldots, N,
$$

where $X_{i}=\frac{\partial}{\partial u_{i, y}}$. It follows from (2.2) that the commutator $Y_{i}=\left[X_{i}, Y\right]=X_{i} Y-Y X_{i}$ of operators $Y$ and $X_{i}, i=0,1, \ldots, N$ also annihilates $I$. In the case of lattice 1.2 operator $Y$ can be represented as:

$$
Y=\sum_{i=0}^{N} u_{i, y} Y_{i}+R
$$

where $Y_{i}$ and $R$ are defined as

$$
\begin{aligned}
Y_{i} & =\frac{\partial}{\partial u_{i}}+X_{i}\left(f_{i}\right) \frac{\partial}{\partial u_{i, x}}+X_{i}\left(D_{x} f_{i}\right) \frac{\partial}{\partial u_{i, x x}}+\cdots \\
& =\frac{\partial}{\partial u_{i}}+r_{i} \frac{\partial}{\partial u_{i, x}}+\left(D_{x}\left(r_{i}\right)+r_{i}^{2}\right) \frac{\partial}{\partial u_{i, x x}}+\cdots \\
R & =\sum_{i=0}^{N}\left(u_{i, x} p_{i}+q_{i}\right) \frac{\partial}{\partial u_{i, x}}+\left(D_{x}\left(u_{i, x} p_{i}+q_{i}\right)+\left(u_{i, x} p_{i}+q_{i}\right) r_{i}\right) \frac{\partial}{\partial u_{i, x x}}+\cdots
\end{aligned}
$$

Let $\mathbf{F}$ be a ring of locally analytical functions of the dynamical variables $\mathbf{u}, \mathbf{u}_{x}, \mathbf{u}_{x x}, \ldots$ We consider the Lie-Rinehart algebra $\mathcal{L}(y, N)$ over the ring $\mathbf{F}$ generated by differential operators $Y, Y_{0}, Y_{1}, \ldots, Y_{N}$. We call this algebra the characteristic Lie algebra of system (2.1) along the $y$-direction. We shall show that we can multiply the elements in the algebra by functions depending on finitely many dynamical variables; this fact distinguishes our algebra from an ordinary Lie algebra. The characteristic Lie algebra of system (2.1) along the $x$-direction is defined in the same way.

Now we shall work with the operators in the algebra $\mathcal{L}(y, N)$. Algebra $\mathcal{L}(y, N)$ is of a finite dimension if there exist a finite basis $Z_{1}, Z_{2}, \ldots, Z_{k}$ consisting of linearly independent operators such that each element $Z \in \mathcal{L}(y, N)$ is represented as a linear combination $Z=a_{1} Z_{1}+a_{2} Z_{2}+\ldots+a_{k} Z_{k}$, where the coefficients $a_{1}, a_{2}, \ldots, a_{k}$ are analytic functions depending on the dynamical variables defined in an open domain. Then the identity $a_{1} Z_{1}+a_{2} Z_{2}+\ldots+a_{k} Z_{k}=0$ implies that $a_{1}=a_{2}=\ldots=a_{k}=0$. System (2.1) is integrable in the sense of Darboux if and only if the characteristic Lie algebras in both directions are of a finite dimension [14].

In our study, we shall apply the operator $D_{x}$ to smooth functions depending on the dynamical variables $\mathbf{u}, \mathbf{u}_{x}, \mathbf{u}_{x x}, \ldots$. On this class of functions, we obtain the following commutation relations for the operators $Y_{i}, R$ :

$$
\begin{aligned}
& {\left[D_{x}, Y_{i}\right]=-r_{i} Y_{i},} \\
& {\left[D_{x}, R\right]=-\sum_{i=0}^{N}\left(u_{i, x} p_{i}+q_{i}\right) Y_{i} .}
\end{aligned}
$$

The following statement holds [13, 19, 14]: 
Lemma 2.1. If a vector field

$$
Z=\sum_{i} z_{1, i} \frac{\partial}{\partial u_{i, x}}+z_{2, i} \frac{\partial}{\partial u_{i, x x}}+\cdots
$$

solves the equation $\left[D_{x}, Z\right]=0$, then $Z=0$.

We shall also use the standard notation $\operatorname{ad}_{X}(Z):=[X, Z]$.

The key method, on which the classification algorithm is based, is a test sequence method. We call a sequence of operators $W_{0}, W_{1}, W_{2}, \ldots$ in the algebra $\mathcal{L}(y, N)$ a test sequence if

$$
\left[D_{x}, W_{m}\right]=\sum_{j=0}^{m} w_{j, m} W_{j}
$$

holds true for all $m$. The test sequence allows us to derive integrability conditions for hyperbolic type system (2.1), see [24, 14, 15].

The first step of our study is to define the functions $p_{n}, r_{n}$. Let us note that when we search the function $r_{n}$ we study the subalgebra Lie generated by the operators $Y_{i}$, see (2.4). It follows from (2.3), (2.4), (2.5) that this subalgebra coincides with the Lie algebra of a hyperbolic type system corresponding the lattice

$$
u_{n, x y}=r_{n}\left(u_{n+1}, u_{n}, u_{n-1}\right) u_{n, y} .
$$

The following statement holds true for this lattice.

Lemma 2.2. If lattice (2.6) is integrable in the sense of Definition 1.1, then it is reduced by point transformations to one of the following forms:

$$
\begin{aligned}
& u_{n, x y}=\left(e^{u_{n}-u_{n-1}}-e^{u_{n+1}-u_{n}}\right) u_{n, y}, \\
& u_{n, x y}=\left(-u_{n+1}+2 u_{n}-u_{n-1}\right) u_{n, y} .
\end{aligned}
$$

Proof of Lemma 2.2 is given in Section 3.

Remark 2.1. If the function $r_{n}$ depends only on the variable $u_{n}$, that is $r_{n}=r_{n}\left(u_{n}\right)$, then

$$
\left[Y_{k}, Y_{j}\right]=0
$$

for all $k, j$ and system (2.6) splits into the system of independent equations $u_{n, x y}=r_{n}\left(u_{n}\right) u_{n, y}$. This system has integrals in the direction we consider. We mention that a wide class of scalar equations of the form $u_{x, y}=f\left(u, u_{x}, u_{x}\right)$ was studied in [14] within the characteristic Lie algebras approach. But the case $r_{n}=r_{n}\left(u_{n}\right)$ or $p_{n}=p_{n}\left(u_{n}\right)$ holds for lattice (1.2) and is to be studied, see Section 4.

\section{INTEGRABILITY CONDITIONS}

3.1. The first test sequence. Let us define a sequence of operators in the characteristic algebra $\mathcal{L}(y, N)$ by the reccurent formula:

$$
Y_{0}, \quad Y_{1}, \quad W_{1}=\left[Y_{0}, Y_{1}\right], \quad W_{2}=\left[Y_{0}, W_{1}\right], \quad \ldots \quad W_{k+1}=\left[Y_{0}, W_{k}\right], \quad \ldots
$$

The following commutation relations are valid for the first elements of the sequence (3.1), see formula 2.5):

$$
\left[D_{x}, Y_{0}\right]=-r_{0} Y_{0}, \quad\left[D_{x}, Y_{1}\right]=-r_{1} Y_{1}
$$


By using the Jacobi identity we get the formulae

$$
\begin{aligned}
{\left[D_{x}, W_{1}\right]=} & -\left(r_{1}+r_{0}\right) W_{1}-Y_{0}\left(r_{1}\right) Y_{1}+Y_{1}\left(r_{0}\right) Y_{0} \\
{\left[D_{x}, W_{2}\right]=} & -\left(r_{1}+2 r_{0}\right) W_{2}-Y_{0}\left(2 r_{1}+r_{0}\right) W_{1}-Y_{0}^{2}\left(r_{1}\right) Y_{1}+Y_{0} Y_{1}\left(r_{0}\right) Y_{0}, \\
{\left[D_{x}, W_{3}\right]=} & -\left(r_{1}+3 r_{0}\right) W_{3}-Y_{0}\left(3 r_{1}+3 r_{0}\right) W_{2}-Y_{0}^{2}\left(3 r_{1}+r_{0}\right) W_{1} \\
& -Y_{0}^{3}\left(r_{1}\right) Y_{1}+Y_{0}^{2} Y_{1}\left(r_{0}\right) Y_{0}, \\
{\left[D_{x}, W_{4}\right]=} & -\left(r_{1}+4 r_{0}\right) W_{4}-Y_{0}\left(4 r_{1}+6 r_{0}\right) W_{3}-Y_{0}^{2}\left(6 r_{1}+4 r_{0}\right) W_{2} \\
& -Y_{0}^{3}\left(4 r_{1}+r_{0}\right) W_{1}-Y_{0}^{4}\left(r_{1}\right) Y_{1}+Y_{0}^{3} Y_{1}\left(r_{1}\right) Y_{0} .
\end{aligned}
$$

It can be proved by induction that (3.1) is a test sequence. Moreover, for $k \geqslant 4$

$$
\left[D_{x}, W_{k}\right]=a_{k} W_{k}+b_{k} W_{k-1}+s_{k} W_{k-2}+t_{k} W_{k-3}+\cdots,
$$

where

$$
\begin{aligned}
& a_{k}=-\left(r_{1}+k r_{0}\right), \quad b_{k}=\frac{k-k^{2}}{2} Y_{0}\left(r_{0}\right)-Y_{0}\left(r_{1}\right) k, \\
& s_{k}=-Y_{0}^{2}\left(3 r_{1}+r_{0}\right)+\frac{1}{2}(k-3) Y_{0}\left(q_{3}+q_{k-1}\right), \\
& t_{k}=-Y_{0}^{3}\left(4 r_{1}+r_{0}\right)+\frac{1}{2}(k-4) Y_{0}\left(s_{4}+s_{k-1}\right) .
\end{aligned}
$$

By assumption, in the algebra $\mathcal{L}(y, N)$ there are finitely many linearly independent elements of sequence (3.1). Therefore, there exists $M$ such that

$$
W_{M}=\lambda W_{M-1}+\cdots,
$$

the operators $Y_{0}, Y_{1}, W_{1}, \ldots, W_{M-1}$ are linearly independent, the dots stand for a linear combination of the operators $Y_{0}, Y_{1}, W_{1}, \ldots, W_{M-2}$.

Let us consider the first three elements.

Lemma 3.1. If condition (1.4) holds, then the operators $Y_{0}, Y_{1}, W_{1}$ are linear independent. Otherwise, if $r_{0}=r_{0}\left(u_{0}\right)$ depends only on the variable $u_{0}$, then $W_{1}=0$.

Proof. Let $r_{0}$ depend on at least one of the variables $u_{-1}, u_{1}$. We are going to prove that $Y_{0}$, $Y_{1}, W_{1}$ are linear independent in this case. We argue by contradiction assuming that

$$
\lambda_{1} W_{1}+\mu_{1} Y_{1}+\mu_{0} Y_{0}=0 .
$$

The operators $Y_{0}, Y_{1}$ are of the form

$$
Y_{0}=\frac{\partial}{\partial u_{0}}+\cdots, \quad Y_{1}=\frac{\partial}{\partial u_{1}}+\cdots,
$$

while $W_{1}$ contains terms of the form $\frac{\partial}{\partial u_{0}}$ and $\frac{\partial}{\partial u_{1}}$. Hence, the coefficients $\mu_{1}, \mu_{0}$ are equal to zero. If $\lambda_{1} \neq 0$, then $W_{1}=0$. We apply the operator $\operatorname{ad}_{D_{x}}$ to both sides of the last identity, then by virtue of $(3.2)$ we obtain the equation

$$
Y_{0}\left(r_{1}\right) Y_{1}-Y_{1}\left(r_{0}\right) Y_{0}=0 .
$$

It implies that $Y_{0}\left(r_{1}\right)=r_{1, u_{0}}=0$ and $Y_{1}\left(r_{0}\right)=r_{0, u_{1}}=0$. This is equivalent to $r_{0, u_{-1}}=0$, $r_{0, u_{1}}=0$ and we arrive at a contradiction to condition 1.4.

By direct calculation of the operator

$$
W_{1}=\left[Y_{0}, Y_{1}\right]=Y_{0} Y_{1}-Y_{1} Y_{0}
$$

and using formula (2.4), we prove the second part of the lemma. The proof is complete.

In what follows we assume that $M \geqslant 2$ and condition (1.4) holds. 
Lemma 3.2. If relation (3.9) holds true for $M \geqslant 2$, then the function $r_{0}$ has one of the following forms:

i) if $\lambda=0$, then

$$
r_{0}\left(u_{1}, u_{0}, u_{-1}\right)=\alpha\left(u_{-1}\right)-\frac{2}{M-1} \alpha\left(u_{0}\right)+\delta\left(u_{1}\right)
$$

ii) if $\lambda \neq 0$, then

$$
r_{0}\left(u_{1}, u_{0}, u_{-1}\right)=\beta\left(u_{-1}\right) e^{-\frac{2}{M(M-1)} \lambda u_{0}}+\psi\left(u_{0}, u_{1}\right),
$$

where functions $\beta$ and $\psi$ satisfy the equation

$$
\lambda \psi\left(u_{0}, u_{1}\right)+\frac{1}{2} M(M-1) \psi_{u_{0}}\left(u_{0}, u_{1}\right)+M e^{-\frac{2}{M(M-1)} \lambda u_{1}} \beta^{\prime}\left(u_{0}\right)=0 .
$$

Proof. We apply the operator $\operatorname{ad}_{D_{x}}$ to both sides of identity (3.9). Combining the coefficients before $W_{M-1}$, we get the equation:

$$
D_{x}(\lambda)=\lambda\left(a_{M}-a_{M-1}\right)+b_{M} .
$$

We substitute formulae (3.8) into 3.13):

$$
D_{x}(\lambda)=-r_{0} \lambda-\frac{M(M-1)}{2} r_{0, u_{0}}-M r_{1, u_{0}} .
$$

From identity (3.14) it follows that $\lambda$ is a constant and

$$
r_{0} \lambda+\frac{M(M-1)}{2} r_{0, u_{0}}+M r_{1, u_{0}}=0 .
$$

Let us apply the operator $\frac{\partial}{\partial u_{2}}$ to 3.15 :

$$
M r_{1, u_{0} u_{2}}=0 .
$$

This is equivalent to $r_{0, u_{-1} u_{1}}=0$ and, hence,

$$
r_{0}\left(u_{1}, u_{0}, u_{-1}\right)=\varphi\left(u_{-1}, u_{0}\right)+\psi\left(u_{0}, u_{1}\right) .
$$

We substitute function 3.15 into 3.14

$$
\lambda \varphi_{u_{-1}}+\frac{M(M-1)}{2} \varphi_{u_{0} u_{-1}}=0 .
$$

We consider two different cases:

i) $\lambda=0$;

ii) $\lambda \neq 0$.

If i) holds, then $\varphi_{u_{0} u_{-1}}=0$, so that $\varphi\left(u_{-1}, u_{0}\right)=\alpha\left(u_{-1}\right)+\beta\left(u_{0}\right)$ and

$$
r_{0}\left(u_{1}, u_{0}, u_{-1}\right)=\alpha\left(u_{-1}\right)+\beta\left(u_{0}\right)+\psi\left(u_{0}, u_{1}\right) .
$$

We re-denote $\beta+\psi \rightarrow \psi$ and we get

$$
r_{0}\left(u_{1}, u_{0}, u_{-1}\right)=\alpha\left(u_{-1}\right)+\psi\left(u_{0}, u_{1}\right) .
$$

We substitute (3.18) and $\lambda=0$ into (3.15):

$$
\frac{M(M-1)}{2} \psi_{u_{0}}\left(u_{0}, u_{1}\right)+M \alpha^{\prime}\left(u_{0}\right)=0 .
$$

Applying the operator $\frac{\partial}{\partial u_{1}}$ to identity 3.19 , we obtain $\psi_{u_{0} u_{1}}=0$ and, hence,

$$
\psi\left(u_{0}, u_{1}\right)=\gamma\left(u_{0}\right)+\delta\left(u_{1}\right) .
$$

We substitute $\psi$ into (3.19) and we find

$$
\gamma\left(u_{0}\right)=-\frac{2}{M-1} \alpha\left(u_{0}\right)+c_{1}
$$


and, then

$$
r_{0}\left(u_{1}, u_{0}, u_{-1}\right)=\alpha\left(u_{-1}\right)-\frac{2}{M-1} \alpha\left(u_{0}\right)+\delta\left(u_{1}\right)
$$

Let us consider case ii). Solution of equation (3.17) is the function

$$
\varphi\left(u_{-1}, u_{0}\right)=\alpha\left(u_{0}\right)+e^{-\frac{2}{M(M-1)} \lambda u_{0}} \beta\left(u_{-1}\right) .
$$

Then function $(3.16)$ becomes

$$
r_{0}\left(u_{1}, u_{0}, u_{-1}\right)=\alpha\left(u_{0}\right)+e^{-\frac{2}{M(M-1)} \lambda u_{0}} \beta\left(u_{-1}\right)+\psi\left(u_{0}, u_{1}\right) .
$$

We redenote $\alpha+\psi \rightarrow \psi$ and we get

$$
r_{0}\left(u_{1}, u_{0}, u_{-1}\right)=e^{-\frac{2}{M(M-1)} \lambda u_{0}} \beta\left(u_{-1}\right)+\psi\left(u_{0}, u_{1}\right) .
$$

Substituting $r_{0}$ into (3.15), we obtain (3.12). The proof is complete.

3.2. Second test sequence. We construct the test sequence containing operators $Y_{0}, Y_{1}$, $Y_{2}$ and their multiple commutators:

$$
\begin{aligned}
& Z_{0}=Y_{0}, \quad Z_{1}=Y_{1}, \quad Z_{2}=Y_{2}, \quad Z_{3}=\left[Y_{1}, Y_{0}\right], \quad Z_{4}=\left[Y_{2}, Y_{1}\right], \\
& Z_{5}=\left[Y_{2}, Z_{3}\right], \quad Z_{6}=\left[Y_{1}, Z_{3}\right], \quad Z_{7}=\left[Y_{1}, Z_{4}\right], \quad Z_{8}=\left[Y_{1}, Z_{5}\right] .
\end{aligned}
$$

The elements $Z_{m}, m>8$ are defined by the recurrent formula $Z_{m}=\left[Y_{1}, Z_{m-3}\right]$.

The following commutation relations hold:

$$
\begin{aligned}
{\left[D_{x}, Y_{0}\right]=} & -r_{0} Y_{0}, \quad\left[D_{x}, Y_{1}\right]=-r_{1} Y_{1}, \quad\left[D_{x}, Y_{2}\right]=-r_{2} Y_{2} \\
{\left[D_{x}, Z_{3}\right]=} & -\left(r_{1}+r_{0}\right) Z_{3}+Y_{0}\left(r_{1}\right) Y_{1}-Y_{1}\left(r_{0}\right) Y_{0} \\
{\left[D_{x}, Z_{4}\right]=} & -\left(r_{2}+r_{1}\right) Z_{4}+Y_{1}\left(r_{2}\right) Y_{2}-Y_{2}\left(r_{1}\right) Y_{1} \\
{\left[D_{x}, Z_{5}\right]=} & -\left(r_{0}+r_{1}+r_{2}\right) Z_{5}-Y_{2}\left(r_{1}+r_{0}\right) Z_{3}+Y_{0}\left(r_{1}\right) Z_{4} \\
& +Y_{2} Y_{0}\left(r_{1}\right) Y_{1}-Y_{2} Y_{1}\left(r_{0}\right) Y_{0}, \\
{\left[D_{x}, Z_{6}\right]=} & -\left(r_{0}+2 r_{1}\right) Z_{6}-Y_{1}\left(2 r_{0}+r_{1}\right) Z_{3}+Y_{1} Y_{0}\left(r_{1}\right) Y_{1}-Y_{1}^{2}\left(r_{0}\right) Y_{0} \\
{\left[D_{x}, Z_{7}\right]=} & -\left(2 r_{1}+r_{2}\right) Z_{7}-Y_{1}\left(r_{1}+2 r_{2}\right) Z_{4}+Y_{1}^{2}\left(r_{2}\right) Y_{2}-Y_{1} Y_{2}\left(r_{1}\right) Y_{1}, \\
{\left[D_{x}, Z_{8}\right]=} & -\left(r_{0}+2 r_{1}+r_{2}\right) Z_{8}+Y_{0}\left(r_{1}\right) Z_{7}-Y_{2}\left(r_{0}+r_{1}\right) Z_{6}-Y_{1}\left(r_{0}+r_{1}+r_{2}\right) Z_{5} \\
& +Y_{1} Y_{0}\left(r_{1}\right) Z_{4}-Y_{1} Y_{2}\left(r_{1}\right) Z_{3}+Y_{1} Y_{2} Y_{0}\left(r_{1}\right) Y_{1}-Y_{1} Y_{2} Y_{1}\left(r_{0}\right) Y_{0} .
\end{aligned}
$$

We recall that we assume condition (1.4), otherwise, starting with $Z_{3}$, all elements of the sequence vanish.

Lemma 3.3. The operators $Z_{0}, Z_{1}, \ldots, Z_{5}$ are linearly independent.

Proof. It is easy to show that the operators $Z_{0}, Z_{1}, \ldots, Z_{4}$ are linearly independent; this is similar to the proof of Lemma 3.1. We prove Lemma 3.3 by arguing by contradiction. We suppose that

$$
Z_{5}=\sum_{j=0}^{4} \lambda_{j} Z_{j}
$$


We apply the operator $\operatorname{ad}_{D_{x}}$ to both sides of identity (3.25), and we use formulae (3.21) - (3.23) to simplify the obtained identity:

$$
\begin{aligned}
-\left(r_{0}+r_{1}+r_{2}\right) & \sum_{j=0}^{4} \lambda_{j} Z_{j}+Y_{0}\left(r_{1}\right) Z_{4}-Y_{2}\left(r_{1}\right) Z_{3}+Y_{2} Y_{0}\left(r_{1}\right) Y_{1}-Y_{2} Y_{1}\left(r_{0}\right) Y_{0} \\
= & \sum_{j=0}^{4} D_{x}\left(\lambda_{j}\right) Z_{j}+\lambda_{4}\left(-\left(r_{2}+r_{1}\right) Z_{4}+Y_{1}\left(r_{2}\right) Y_{2}-Y_{2}\left(r_{1}\right) Y_{1}\right) \\
& +\lambda_{3}\left(-\left(r_{1}+r_{0}\right) Z_{3}+Y_{0}\left(r_{1}\right) Y_{1}-Y_{1}\left(r_{0}\right) Y_{0}\right) \\
& -\lambda_{2} r_{2} Y-2-\lambda_{1} r_{1} Y_{1}-\lambda_{0} r_{0} Y_{0} .
\end{aligned}
$$

Combining the coefficients at $Z_{4}$ in $(3.26)$, we get the equation:

$$
D_{x}\left(\lambda_{4}\right)=-r_{0} \lambda_{4}+r_{1, u_{0}} .
$$

This identity implies that $\lambda_{4}$ is a constant and

$$
-r_{0} \lambda_{4}+r_{1, u_{0}}=0
$$

We shall study this equation in two different cases i) and ii):

i) If $r_{0}$ is defined by formula (3.10), then 3.27 casts into the form

$$
-\left(\alpha\left(u_{-1}\right)-\frac{2}{M-1} \alpha\left(u_{0}\right)+\delta\left(u_{1}\right)\right) \lambda_{4}+\alpha^{\prime}\left(u_{0}\right)=0 .
$$

If $\lambda_{4} \neq 0$, then (3.28) implies that functions $\alpha, \delta$ are constants since the variables $u_{1}, u_{0}, u_{-1}$ are independent. Then we get that $r_{0}$ is a constant that contradicts condition (1.4). If $\lambda_{4}=0$, then it follows from $(3.28)$ that $\alpha^{\prime}\left(u_{0}\right)=0$ and

$$
r_{0}\left(u_{1}, u_{0}, u_{-1}\right)=\delta\left(u_{1}\right) .
$$

ii) If $r_{0}$ is defined by (3.11), then identity (3.27) becomes

$$
-\left(\beta\left(u_{-1}\right) e^{-\frac{2}{M(M-1) \lambda u_{0}}}+\psi\left(u_{0}, u_{1}\right)\right) \lambda_{4}+\beta^{\prime}\left(u_{0}\right) e^{-\frac{2}{M(M-1)} \lambda u_{1}}=0 .
$$

We apply the operator $\frac{\partial}{\partial u_{-1}}$ to 3.30 :

$$
\beta^{\prime}\left(u_{-1}\right) e^{-\frac{2}{M(M-1)} \lambda u_{0}} \lambda_{4}=0 .
$$

If $\lambda_{4}=0$, then it follows from 3.30 that $\beta^{\prime}\left(u_{0}\right)=0$ and, hence, $\beta=c_{4}$, where $c_{4}$ is a constant. If $\lambda_{4} \neq 0$, then it follows from (3.30) that

$$
\beta\left(u_{-1}\right) e^{-\frac{2}{M(M-1) \lambda u_{0}}}+\psi\left(u_{0}, u_{1}\right)=0 .
$$

The expression in the left hand side of the last identity coincides exactly with $r_{0}\left(u_{1}, u_{0}, u_{-1}\right)$. Therefore, the last identity contradicts condition (1.4).

Thus, we obtain that $\lambda_{4}=0$ and $r_{0}$ is defined by the formula

$$
r_{0}\left(u_{1}, u_{0}, u_{-1}\right)=c_{4} e^{-\frac{2}{M(M-1)} \lambda u_{0}}+\psi\left(u_{0}, u_{1}\right) .
$$

We collect the coefficients at $Z_{3}$ in (3.26), take into consideration that $\lambda_{4}=0$, and we obtain the equation

Hence, $\lambda_{3}$ is a constant, and

$$
D_{x}\left(\lambda_{3}\right)=-r_{2} \lambda_{3}-r_{1, u_{2}}
$$

$$
r_{2} \lambda_{3}+r_{1, u_{2}}=0 .
$$

Applying the shift operator, we get the equation

$$
r_{1} \lambda_{3}+r_{0, u_{1}}=0 .
$$


i) Let us substitute the function $r_{0}$ defined by formula (3.29) into (3.32):

$$
\delta\left(u_{2}\right) \lambda_{3}+\delta^{\prime}\left(u_{1}\right)=0 .
$$

A simple analysis of the last equation gives the contradiction to condition (1.4).

ii) Let us substitute the function $r_{0}$ defined by formula (3.31) into $(3.32)$ :

$$
\left(c_{4} e^{-\frac{2}{M(M-1)} \lambda u_{1}}+\psi\left(u_{1}, u_{2}\right)\right) \lambda_{3}+\psi_{u_{1}}\left(u_{0}, u_{1}\right)=0 .
$$

We apply the operator $\frac{\partial}{\partial u_{2}}$ to both sides of identity (3.33) $\psi_{u_{2}}\left(u_{1}, u_{2}\right) \lambda_{3}=0$. Studying 3.33 in this case, we arrive to contradiction to condition (1.4).

Otherwise, if $\lambda_{3} \neq 0$, then the expression in the left hand side of identity (3.33), coinciding with $r_{1}$, is equal to zero. Thus, we obtain the contradiction to condition (1.4). The proof is complete.

For further purposes, it is convenient to divide sequence 3.26 into three subsequences $\left\{Z_{3 m}\right\}$, $\left\{Z_{3 m+1}\right\},\left\{Z_{3 m+2}\right\}$

Lemma 3.4. Operator $\operatorname{ad}_{\mathrm{D}_{\mathrm{x}}}$ acts on sequence (3.26) according the following formulae:

$$
\begin{aligned}
{\left[D_{x}, Z_{3 m}\right]=} & -\left(r_{0}+m r_{1}\right) Z_{3 m}+\left(\frac{m-m^{2}}{2} Y_{1}\left(r_{1}\right)-m Y_{1}\left(r_{0}\right)\right) Z_{3 m-3}+\cdots \\
{\left[D_{x}, Z_{3 m+1}\right]=} & -\left(r_{2}+m r_{1}\right) Z_{3 m+1}+\left(\frac{m-m^{2}}{2} Y_{1}\left(r_{1}\right)-m Y_{1}\left(r_{2}\right)\right) Z_{3 m-2}+\cdots, \\
{\left[D_{x}, Z_{3 m+2}\right]=} & -\left(r_{0}+m r_{1}+r_{2}\right) Z_{3 m+2}+Y_{0}\left(r_{1}\right) Z_{3 m+1}-Y_{2}\left(r_{1}\right) Z_{3 m} \\
& -(m-1)\left(\frac{m}{2} Y_{1}\left(r_{1}\right)+Y_{1}\left(r_{0}+r_{2}\right)\right) Z_{3 m-1}+\cdots
\end{aligned}
$$

Lemma 3.4 can be easily proved by induction.

Theorem 3.1. Assume that $Z_{3 k+2}$ is a linear combination

$$
Z_{3 k+2}=\lambda_{k} Z_{3 k+1}+\mu_{k} Z_{3 k}+\nu_{k} Z_{3 k-1}+\cdots
$$

of the previous terms in sequence (3.26) and none of the operators $Z_{3 j+2}$ for $j<k$ is a linear combination of operators $Z_{s}$ with $s<3 j+2$. Then the coefficient $\nu_{k}$ satisfies the equation

$$
D_{x}\left(\nu_{k}\right)=-r_{1} \nu_{k}-\frac{k(k-1)}{2} Y_{1}\left(r_{1}\right)-(k-1) Y_{1}\left(r_{0}+r_{2}\right) .
$$

Lemma 3.5. Suppose that the assumptions of Theorem 3.1 are satisfied and the operator $Z_{3 k}$ (the operator $Z_{3 k+1}$ ) is linearly expressed in terms of the operators $Z_{i}, i<3 k$. Then in this decomposition the coefficient at $Z_{3 k-1}$ vanishes.

Proof. We argue by contradiction. Suppose that

$$
Z_{3 k}=\lambda Z_{3 k-1}+\cdots
$$

and $\lambda \neq 0$. We apply the operator $\operatorname{ad}_{\mathrm{D}_{\mathrm{x}}}$ to both sides of identity (3.36). Using formulae from Lemma 3.4 , we get

$$
-\left(r_{0}+k r_{1}\right) \lambda Z_{3 k-1}+\cdots=D_{x}(\lambda) Z_{3 k-1}-\lambda\left(r_{0}+(k-1) r_{1}+r_{2}\right) Z_{3 k-1}+\cdots
$$

Collecting coefficients at $Z_{3 k-1}$, we obtain

$$
D_{x}(\lambda)=\lambda\left(r_{2}-r_{1}\right) k .
$$

This equation implies that $\lambda$ is a constant and $\lambda\left(r_{2}-r_{1}\right) k=0$. Then $r_{2}=r_{1}=$ const that contradictions condition (1.4). The proof is complete. 
In order to prove Theorem 3.1, we apply the operator $\operatorname{ad}_{D_{x}}$ to both sides of the identity (3.34). Then we simplify a obtained identity using formulae from Lemma 3.4. Collecting coefficients at $Z_{3 k-1}$, we obtain equation (3.35).

The next step of our work is studying equation (3.35) as $r_{0}$ is defined by formulae (3.10) or (3.11) under condition (1.4) and for $M \geqslant 2, k \geqslant 2$.

We find exact values of coefficients in equation (3.35) and substitute them into (3.35):

$$
D_{x}\left(\nu_{k}\right)=-r_{1} \nu_{k}-\frac{k(k-1)}{2} r_{1, u_{1}}-(k-1)\left(r_{0, u_{1}}+r_{2, u_{1}}\right) .
$$

This equation implies that $\nu_{k}$ is a constant and, hence,

$$
\nu_{k} r_{1}+\frac{k(k-1)}{2} r_{1, u_{1}}+(k-1)\left(r_{0, u_{1}}+r_{2, u_{1}}\right)=0
$$

Lemma 3.6. If relations (3.9), 3.34) hold true for some $M \geqslant 2, k \geqslant 2$, and condition (1.4) holds true, then

i) if $\lambda=0, \nu_{k}=0$, then

$$
r_{n}\left(u_{n+1}, u_{n}, u_{n-1}\right)=\alpha\left(u_{n-1}\right)-\frac{2}{M-1} \alpha\left(u_{n}\right)+\left(\frac{k}{M-1}-1\right) \alpha\left(u_{n+1}\right)+c_{1} ;
$$

ii) if $\lambda \neq 0, \nu_{k}=0, k=2, M \neq 3$, then

$$
r_{n}\left(u_{n+1}, u_{n}, u_{n-1}\right)=e^{u_{n}-h u_{n-1}}+c e^{u_{n+1}-h u_{n}} ;
$$

iii) if $\lambda \neq 0, \nu_{k} \neq 0$, then $r_{n}$ is defined by formula (3.39).

The proof of this lemma is rather complicated and is presented in Appendix.

We proceed to relations (3.9), (3.34).

We need another one test sequence:

$$
Y_{0}, \quad Y_{1}, \quad \bar{W}_{1}=\left[Y_{1}, Y_{0}\right], \quad \bar{W}_{2}=\left[Y_{1}, \bar{W}_{1}\right], \ldots \bar{W}_{k+1}=\left[Y_{1}, \bar{W}_{k}\right] \ldots
$$

The following commutation relation hold:

$$
\begin{aligned}
{\left[D_{x}, \bar{W}_{1}\right]=} & -\left(r_{1}+r_{0}\right) \bar{W}_{1}+Y_{0}\left(r_{1}\right) Y_{1}-Y_{1}\left(r_{0}\right) Y_{0} \\
{\left[D_{x}, \bar{W}_{2}\right]=} & -\left(2 r_{1}+r_{0}\right) \bar{W}_{2}-Y_{1}\left(r_{1}+2 r_{0}\right) \bar{W}_{1}+Y_{1} Y_{0}\left(r_{1}\right) Y_{1}-Y_{1}^{2}\left(r_{0}\right) Y_{0}, \\
{\left[D_{x}, \bar{W}_{3}\right]=} & -\left(3 r_{1}+r_{0}\right) \bar{W}_{3}-Y_{1}\left(3 r_{1}+3 r_{0}\right) \bar{W}_{2}-Y_{1}^{2}\left(r_{1}+3 r_{0}\right) \bar{W}_{1} \\
& +Y_{1}^{2} Y_{0}\left(r_{1}\right) Y_{1}-Y_{1}^{3}\left(r_{0}\right) Y_{0} \\
{\left[D_{x}, \bar{W}_{4}\right]=} & -\left(4 r_{1}+r_{0}\right) \bar{W}_{4}-Y_{1}\left(6 r_{1}+4 r_{0}\right) \bar{W}_{3}-Y_{1}^{2}\left(4 r_{1}+6 r_{0}\right) \bar{W}_{2} \\
& -Y_{1}^{3}\left(r_{1}+4 r_{0}\right) \bar{W}_{1}+Y_{1}^{3} Y_{0}\left(r_{1}\right) Y_{1}-Y_{1}^{4}\left(r_{0}\right) Y_{0}
\end{aligned}
$$

It is easy to prove that

$$
\left[D_{x}, \bar{W}_{k}\right]=\bar{a}_{k} \bar{W}_{k}+\bar{b}_{k} \bar{W}_{k-1}+\bar{s}_{k} \bar{W}_{k-2}+\cdots
$$

for $k \geqslant 3$, where

$$
\begin{aligned}
& \bar{a}_{k}=-\left(k r_{1}+r_{0}\right), \quad \bar{b}_{k}=\frac{k-k^{2}}{2} Y_{1}\left(r_{1}\right)-Y_{1}\left(r_{0}\right) k, \\
& \bar{s}_{k}=-Y_{1}^{2}\left(r_{1}+3 r_{0}\right)+\frac{1}{2}(k-3) Y_{1}\left(\bar{q}_{3}+\bar{q}_{k-1}\right) .
\end{aligned}
$$

We observe that the first terms $Y_{0}, Y_{1}, \bar{W}_{1}=-W_{1}$ obey Lemma 3.1 .

We suppose that $\mathcal{L}(y, N)$ is finitely-dimensional, that is, each sequence of its elements terminates at some step. Consequently, there exists $N$ such that:

$$
\bar{W}_{N}=\Lambda \bar{W}_{N-1}+\cdots \text {, }
$$

where the operators $Y_{0}, Y_{1}, \bar{W}_{1}, \ldots, \bar{W}_{N-1}$ are linearly independent, and the dots stand for linear combination of the operators $Y_{0}, Y_{1}, \bar{W}_{1}, \ldots, \bar{W}_{N-2}$. 
3.3. Case $M=2$. Suppose that relation $(3.9)$ holds true for $M=2$ :

$$
W_{2}=\lambda W_{1}+\varepsilon Y_{1}+\eta Y_{0} .
$$

We apply the operator $\operatorname{ad}_{\mathrm{D}_{\mathrm{x}}}$ to both sides of identity $(3.46)$ and we get:

$$
\begin{aligned}
-\left(r_{1}+2 r_{0}\right)\left(\lambda W_{1}+\varepsilon Y_{1}+\eta Y_{0}\right) & -Y_{0}\left(2 r_{1}+r_{0}\right) W_{1}-Y_{0}^{2}\left(r_{1}\right) Y_{1}+Y_{0} Y_{1}\left(r_{0}\right) Y_{0} \\
& =\lambda\left(-\left(r_{1}+r_{0}\right) W_{1}-Y_{0}\left(r_{1}\right) Y_{1}+Y_{1}\left(r_{0}\right) Y_{0}\right)-\varepsilon r_{1} Y_{1}-\eta r_{0} Y_{0} .
\end{aligned}
$$

Collecting the coefficients at independent operators $W_{1}, Y_{1}, Y_{0}$, we obtain the system

$$
\begin{aligned}
& r_{0} \lambda+2 r_{1, u_{0}}+r_{0, u_{0}}=0, \\
& 2 r_{0} \varepsilon+r_{1, u_{0} u_{0}}-\lambda r_{1, u_{0}}=0, \\
- & \left(r_{1}+r_{0}\right) \eta+r_{0, u_{0} u_{1}}-\lambda r_{0, u_{1}}=0 .
\end{aligned}
$$

3.3.i) Let us consider the case when thefunction $r_{n}$ is described by formula (3.38) and $\lambda=0$. We substitute function (3.38) and $\lambda=0$ into system (3.47)-(3.49). Then equation (3.47) becomes identity and we arrive to the system:

$$
\begin{aligned}
& 2\left(\alpha\left(u_{-1}\right)-2 \alpha\left(u_{0}\right)+(k-1) \alpha\left(u_{1}\right)+c_{1}\right) \varepsilon+\frac{d^{2} \alpha\left(u_{0}\right)}{d u_{0}^{2}}=0, \\
& \left(\alpha\left(u_{0}\right)+2 \alpha\left(u_{1}\right)-(k-1) \alpha\left(u_{2}\right)+2 c_{1}+\alpha\left(u_{-1}\right)+(k-1) \alpha\left(u_{1}\right)\right) \eta=0 .
\end{aligned}
$$

This system yields that

$$
\begin{aligned}
& \varepsilon=\eta=0, \quad \alpha\left(u_{0}\right)=C_{1} u_{0}+C_{2}, \\
& r_{0}\left(u_{1}, u_{0}, u_{-1}\right)=(k-1) C_{1} u_{1}-2 C_{1} u_{0}+C_{1} u_{-1}+C_{3},
\end{aligned}
$$

where $C_{3}=-2 C_{2}+k C_{2}+c_{1}$. We will study the lattice corresponding to this function, in Section 3.5.i, see (3.62).

3.3.ii) Let us consider the case when the function $r_{n}$ is described by formula $(3.39)$ and $\lambda \neq 0$. System (3.47)-(3.49) casts into the form:

$$
\begin{aligned}
& (\lambda+1) e^{u_{0}-h u_{-1}}+(-c h+\lambda c-2 h) e^{u_{1}-h u_{0}}=0, \\
& 2 \varepsilon e^{u_{0}-h u_{-1}}+\left(2 \varepsilon c+h^{2}+\lambda h\right) e^{u_{1}-h u_{0}}=0, \\
& -\eta e^{u_{0}-h u_{-1}}+(-\eta-\eta c-c h-\lambda c) e^{u_{1}-h u_{0}}-\eta c e^{u_{2}-h u_{1}}=0 .
\end{aligned}
$$

A simple analysis of the last system leads us to the identities $\lambda=-1, h=1, c=-1, \varepsilon=\eta=0$. We get that $r_{n}$ has the following form:

$$
r_{n}\left(u_{n+1}, u_{n}, u_{n-1}\right)=e^{u_{n}-u_{n-1}}-e^{u_{n+1}-u_{n}} .
$$

And $W_{2}=-W_{1}, \bar{W}_{2}=\bar{W}_{1}$.

Now let us substitute 3.50 into 3.37):

$$
\left(\nu_{k}+\frac{1}{2} k^{2}-\frac{3}{2} k+1\right) e^{u_{1}-u_{0}}+\left(-\nu_{k}+\frac{1}{2} k^{2}-\frac{3}{2} k+1\right) e^{u_{2}-u_{1}}=0,
$$

which implies $\nu_{k}=0, k=2$.

Thus, relation (3.34) is of the form

$$
Z_{8}=\rho Z_{4}+\sigma Z_{3}+\tau Z_{2}+\phi Z_{1}+\pi Z_{0}
$$

and

$$
\begin{aligned}
& Z_{6}=\left[Y_{1},\left[Y_{1}, Y_{0}\right]\right]=\bar{W}_{2}=\bar{W}_{1}=\left[Y_{1}, Y_{0}\right]=Z_{3}, \\
& Z_{7}=\left[Y_{1},\left[Y_{2}, Y_{1}\right]\right]=D_{n}\left[Y_{0},\left[Y_{1}, Y_{0}\right]\right]=-D_{n} W_{2}=D_{n} W_{1}=-Z_{4} .
\end{aligned}
$$


Commutation relation $(3.24)$ become

$$
\begin{aligned}
{\left[D_{x}, Z_{8}\right]=} & -\left(r_{0}+2 r_{1}+r_{2}\right) Z_{8}-Y_{1}\left(r_{0}+r_{1}+r_{2}\right) Z_{5}+\left(Y_{1} Y_{0}\left(r_{1}\right)-Y_{0}\left(r_{1}\right)\right) Z_{4} \\
& -\left(Y_{2}\left(r_{0}+r_{1}\right)+Y_{1} Y_{2}\left(r_{1}\right)\right) Z_{3}+Y_{1} Y_{2} Y_{0}\left(r_{1}\right) Y_{1}-Y_{1} Y_{2} Y_{1}\left(r_{0}\right) Y_{0} .
\end{aligned}
$$

We apply the operator $\operatorname{ad}_{\mathrm{D}_{\mathrm{x}}}$ to both sides of identity (3.51) and take into consideration the formulae (3.20)-(3.23), (3.52), then we collect coefficients at independent operators $Z_{4}, Z_{3}, Z_{2}$, $Z_{1}, Z_{0}$ :

$$
\begin{aligned}
& -\left(e^{u_{0}-u_{-1}}-e^{u_{2}-u_{1}}\right) \rho=0, \quad\left(-e^{u_{3}-u_{2}}+e^{u_{1}-u_{0}}\right) \sigma=0, \\
& -\left(e^{u_{0}-u_{-1}}+e^{u_{1}-u_{0}}-2 e^{u_{2}-u_{1}}\right) \tau+\rho e^{u_{2}-u_{1}}=0, \\
& -\left(e^{u_{0}-u_{-1}}-e^{u_{3}-u_{2}}\right) \phi-\rho e^{u_{2}-u_{1}}+\sigma e^{u_{1}-u_{0}}=0 . \\
& -\left(2 e^{u_{1}-u_{0}}-e^{u_{2}-u_{1}}-e^{u_{3}-u_{2}}\right) \pi-\sigma e^{u_{1}-u_{0}}=0 .
\end{aligned}
$$

It is clear that $\rho=\sigma=\tau=\phi=\pi=0$. Hence, $Z_{8}=0$.

3.4. Case $M=3$. Suppose that relation $(3.9)$ holds true for $M=3$ :

$$
W_{3}=\lambda W_{2}+\rho W_{1}+\varepsilon Y_{1}+\eta Y_{0} .
$$

We apply the operator $\operatorname{ad}_{\mathrm{D}_{\mathrm{x}}}$ to both sides of identity (3.53) and use formulae (3.2), (3.3), (3.4), (3.5). Collecting coefficients at the independent operators, we obtain the system

$$
\begin{aligned}
& r_{0} \lambda+3 r_{1, u_{0}}+3 r_{0, u_{0}}=0, \\
& -2 r_{0} \rho+\lambda\left(2 r_{1, u_{0}}+r_{0, u_{0}}\right)-3 r_{1, u_{0} u_{0}}-r_{0, u_{0} u_{0}}=0, \\
& -3 r_{0} \varepsilon+\lambda r_{1, u_{0} u_{0}}+\rho r_{1, u_{0}}-r_{1, u_{0} u_{0} u_{0}}=0, \\
& -\left(r_{1}+2 r_{0}\right) \eta-\lambda r_{0, u_{0} u_{1}}-\rho r_{0, u_{1}}+r_{0, u_{0} u_{0} u_{1}}=0 .
\end{aligned}
$$

3.4.i) Let us consider case (3.38), $\lambda=0$. It follows from equations (3.54)-(3.57) that

$$
\alpha\left(u_{n}\right)=C_{1} u_{n}+C_{2}, \quad r_{n}\left(u_{n+1}, u_{n}, u_{n-1}\right)=\frac{k-2}{2} C_{1} u_{n+1}-C_{1} u_{n}+C_{1} u_{n-1}+C_{3},
$$

where $C_{3}=\frac{1}{2} C_{2} k-C_{2}+c_{1}$. Further study of the lattice with $r_{n}$ defined by this formula is provided in 3.5.i, see (3.62).

3.4.ii) Let us consider case $(3.39) \lambda \neq 0$. We substitute $r_{n}$ into (3.47)-(3.49). Studying this system, we obtain that $\lambda=-3, \rho=-2, \varepsilon=\eta=0, h=1, c=-\frac{1}{2}$. The function 3.39 becomes

$$
r_{n}\left(u_{n+1}, u_{n}, u_{n-1}\right)=e^{u_{n}-u_{n-1}}-\frac{1}{2} e^{u_{n+1}-u_{n}} .
$$

We substitute this function into equation (3.37) and we get $k=1$ or $k=\frac{5}{2}$. These identities contradict condition $k \geqslant 2$.

3.5. Case $M>3$. Let the following relation be true for $M>3$

$$
W_{M}=\lambda W_{M-1}+\rho W_{M-2}+\kappa W_{M-3}+\cdots
$$

Taking into account formula (3.7), we apply the operator $\operatorname{ad}_{\mathrm{D}_{\mathrm{x}}}$ to both sides of the above identity:

$$
\begin{aligned}
a_{M}\left(\lambda W_{M-1}\right. & \left.+\rho W_{M-2}+\kappa W_{M-3}+\cdots\right)+b_{M} W_{M-1}+s_{M} W_{M-2}+t_{M} W_{M-3}+\cdots \\
= & \lambda\left(a_{M-1} W_{M-1}+b_{M-1} W_{M-2}+s_{M-1} W_{M-3}+\cdots\right) \\
& +\rho\left(a_{M-2} W_{M-2}+b_{M-2} W_{M-3}+\cdots\right)+\kappa\left(a_{M-3} W_{M-3}+\cdots\right)
\end{aligned}
$$

We collect coefficients at the independent operators $W_{M-1}, W_{M-2}, W_{M-3}$ :

$$
\begin{aligned}
& \lambda\left(a_{M}-a_{M-1}\right)+b_{M}=0, \\
& \rho\left(a_{M}-a_{M-2}\right)+s_{M}-\lambda b_{M-1}=0, \\
& \kappa\left(a_{M}-a_{M-3}\right)+t_{M}-\lambda s_{M-1}-\rho b_{M-2}=0 .
\end{aligned}
$$


3.5.i) By system (3.59)-(3.61) we obtain that $\alpha\left(u_{n}\right)=C_{1} u_{n}+C_{2}$ and

$$
r_{n}\left(u_{n+1}, u_{n}, u_{n-1}\right)=\frac{k-(M-1)}{M-1} C_{1} u_{n+1}-\frac{2 C_{1}}{M-1} u_{n}+C_{1} u_{n-1}+C_{3},
$$

where

$$
C_{3}=\frac{c_{1} M-c_{1}-2 C_{2}+k C_{2}}{M-1} .
$$

Now we consider the function

$$
r_{n}\left(u_{n+1}, u_{n}, u_{n-1}\right)=c_{1} u_{n+1}+c_{2} u_{n}+c_{3} u_{n-1}+c_{4} .
$$

Commutation relations (3.7), (3.44) become

$$
\left[D_{x}, W_{k}\right]=a_{k} W_{k}+b_{k} W_{k-1}, \quad\left[D_{x}, \bar{W}_{k}\right]=\bar{a}_{k} \bar{W}_{k}+\bar{b}_{k} \bar{W}_{k-1},
$$

where

$$
\begin{array}{ll}
a_{k}=-\left(r_{1}+k r_{0}\right), & b_{k}=\frac{k-k^{2}}{2} c_{2}-c_{3} k, \\
\bar{a}_{k}=-\left(k r_{1}+r_{0}\right), & \bar{b}_{k}=\frac{k-k^{2}}{2} c_{2}-c_{1} k .
\end{array}
$$

Assume that sequence $\left\{W_{n}\right\}$ is terminated at the step $M$ :

$$
W_{M}=\sum_{k=1}^{M-1} \Lambda_{M-k} W_{M-k}+\phi_{1} Y_{1}+\phi_{0} Y_{0} .
$$

We apply the operator $\operatorname{ad}_{\mathrm{D}_{\mathrm{x}}}$ to both sides of identity 3.66

$$
\begin{aligned}
& a_{M}\left(\sum_{k=1}^{M-1} \Lambda_{M-k} W_{M-k}+\phi_{1} Y_{1}+\phi_{0} Y_{0}\right)+b_{M} W_{M-1} \\
& =\sum_{k=1}^{M-2} \Lambda_{M-k}\left(a_{M-k} W_{M-k}+b_{M-k} W_{M-k-1}\right) \\
& \quad+\Lambda_{1}\left(-\left(r_{1}+r_{0}\right) W_{1}-c_{3} Y_{1}+c_{1} Y_{0}\right)-\phi_{1} r_{1} Y_{1}-\phi_{0} r_{0} Y_{0} .
\end{aligned}
$$

We collect the coefficients at $W_{M-1}$ in this identity:

$$
\Lambda_{M-1}\left(a_{M}-a_{M-1}\right)+b_{M}=0 .
$$

We substitute formulae (3.64), 3.65) into the last equation:

$$
-\Lambda_{M-1}\left(c_{1} u_{1}+c_{2} u_{0}+c_{3} u_{-1}+c_{4}\right)+\frac{M-M^{2}}{2} c_{2}-c_{3} M=0
$$

A simple analysis of this equation shows that $\Lambda_{M-1}=0$ and $c_{3}=\frac{1-M}{2} c_{2}$. Then, collecting coefficients before $W_{M-k}, k=2, \ldots, M-2$, we arrive at the equations

$$
\Lambda_{M-k}\left(a_{M}-a_{M-k}\right)=0, \quad k=2, \ldots, M-2,
$$

which implies $\Lambda_{M-k}=0, k=2, \ldots, M-2$. The coefficient at $W_{1}$ is $\Lambda_{1}\left(a_{M}+r_{1}+r_{0}\right)=0$. Then $\Lambda_{1}=0$. The coefficients at $Y_{1}$ and $Y_{0}$ read as $\left(a_{M}+r_{1}\right) \phi_{1}=0,\left(a_{M}+r_{0}\right) \phi_{0}=0$ and hence, $\phi_{1}=\phi_{0}=0$. Thus, $W_{M}=0$.

Similarly, if sequence $\left\{\bar{W}_{k}\right\}$ is terminated at step $N$, then $c_{1}=\frac{1-N}{2} c_{2}$ and $\bar{W}_{N}=0$. As a result, we obtain:

$$
r_{n}\left(u_{n+1}, u_{n}, u_{n-1}\right)=\frac{1-N}{2} c_{2} u_{n+1}+c_{2} u_{n}+\frac{1-M}{2} c_{2} u_{n-1}+c_{4} .
$$

By rescaling $\frac{c_{2}}{2} u_{i} \rightarrow v_{i}$, the original lattice is reduced to a lattice of the same form with function $r_{n}$ defined by the formula

$$
r_{n}\left(u_{n+1}, u_{n}, u_{n-1}\right)=(1-N) u_{n+1}+2 u_{n}+(1-M) u_{n-1}+c,
$$


where $c$ is an arbitrary constant. If $4-M-N \neq 0$, then we exclude constant $c$ by the shift transformation $u \rightarrow u-\frac{c}{4-M-N}$. If $M+N=4$, then $M=N=2$, and $c$ is excluded by the transformation $u_{n} \rightarrow u_{n}+\frac{c}{2} n^{2}$. Thus, the function $r_{n}$ becomes:

$$
r_{n}\left(u_{n+1}, u_{n}, u_{n-1}\right)=(1-N) u_{n+1}+2 u_{n}+(1-M) u_{n-1}
$$

and, in particular,

$$
r_{n}\left(u_{n+1}, u_{n}, u_{n-1}\right)=-u_{n+1}+2 u_{n}-u_{n-1} .
$$

We substitute (3.67) into (3.37), and we get that $k=M+N-2$. We substitute (3.68) into (3.37), and we get that $k=2$.

Let us consider lattice (2.6) when $r_{n}$ is defined by (3.67). We impose cut-off conditions $u_{0}=0, u_{L+1}=0$ and we reduce this lattice to the following hyperbolic system:

$$
\begin{aligned}
& u_{1, x y}=\left(2 u_{1}+p u_{2}\right) u_{1, y}, \\
& u_{k, x y}=\left(q u_{k-1}+2 u_{k}+p u_{k+1}\right) u_{k, y}, \quad 2 \leqslant k \leqslant L-1, \\
& u_{L, x y}=\left(q u_{L-1}+2 u_{L}\right) u_{L, y},
\end{aligned}
$$

where $p=1-N, q=1-M$; we recall that $N>1, M>1$. This system is reduced by differential substitution $v_{i}=\ln u_{i, y}$ to the exponential system:

$$
\begin{aligned}
& v_{1, x y}=2 e^{v_{1}}+p e^{v_{2}}, \\
& v_{k, x y}=q e^{v_{k-1}}+2 e^{v_{k}}+p e^{v_{k+1}}, \quad 2 \leqslant k \leqslant L-1, \\
& v_{L, x y}=q e^{v_{L-1}}+2 e^{v_{L}} . \\
& \left(\mathbf{v}_{x y}=A e^{\mathbf{v}}\right) .
\end{aligned}
$$

We denote by $A$ the matrix of coefficients before exponents in the right hand side of the system and we denote by $\mathbf{v}=\left(v_{1}, v_{2}, \ldots, v_{K}\right)^{T}, e^{\mathbf{v}}=\left(e^{v_{1}}, e^{v_{2}}, \ldots, e^{v_{K}}\right)^{T}$ the column vectors. System 3.70 is related with the system

$$
\begin{aligned}
& w_{1, x y}=e^{2 w_{1}+p w_{2}}, \\
& w_{k, x y}=e^{q w_{k-1}+2 w_{k}+p w_{k+1}}, \quad 2 \leqslant k \leqslant L-1, \\
& w_{L, x y}=e^{q w_{L-1}+2 w_{L}} . \\
& \left(\mathbf{w}_{x y}=e^{A \mathbf{w}}\right) .
\end{aligned}
$$

by the following point change of variables

$$
\begin{aligned}
& v_{1}=2 w_{1}+p w_{2}, \\
& v_{k}=q w_{k-1}+2 w_{k}+p w_{k+1}, \quad 2 \leqslant k \leqslant L-1, \\
& v_{L}=q w_{L-1}+2 w_{L} . \\
& (\mathbf{v}=A \mathbf{w}) .
\end{aligned}
$$

System (3.71) is reduced to system 3.69 by differential substitution

$$
u_{i}=w_{i, x} .
$$

It is shown in [11, 25] (see also [14]) that if $A$ is the Cartan matrix of a simple Lie algebra, then the system (3.70) $(3.71))$ is integrated in quadratures. Comparing the Cartan matrix and matrix $A$, one can see that $p=q=-1$. Thus, we have that $M=N=2$. In this case we find that $W_{2}=0, \bar{W}_{2}=0, Z_{8}=0$.

Let us show that if systems (3.70), (3.71) is integrable in the sense of Darboux then system (3.69) is integrable in the sense of Darboux, too. Suppose that $\bar{I}\left(\mathbf{w}_{x}, \mathbf{w}_{x x}, \cdots\right)$ is an $y$-integral of system (3.71). We change variables by the rule $w_{i, x}=u_{i}, w_{i, x x}=u_{i, x}$ and so on, due to 
(3.73), then we obtain an $y$-integral $\bar{I}\left(\mathbf{u}, \mathbf{u}_{x}, \cdots\right)$ of system 3.69$)$. Assume that $I\left(\mathbf{v}_{y}, \mathbf{v}_{y y}, \cdots\right)$ is an $x$-integral of system $(3.70)$. Using $(3.73)$ and $(3.72)$, we derive:

$$
\mathbf{u}=\mathbf{w}_{x}=A^{-1} \mathbf{v}_{x} .
$$

Hence, by virtue 3.70

$$
\mathbf{u}_{y}=A^{-1} \mathbf{v}_{x y}=A^{-1} A e^{\mathbf{v}}=e^{\mathbf{v}} .
$$

We change variables in the function $I\left(\mathbf{v}_{y}, \mathbf{v}_{y y}, \ldots\right)$ by the rule $v_{i}=\ln u_{i, y}, v_{i, y}=\left(\ln u_{i, y}\right)_{y}$ and so on. Thus, we get an $x$-integral $I\left(\left(\ln u_{i, y}\right)_{y},\left(\ln u_{i, y}\right)_{y y}, \ldots\right)$ of system $(3.69)$.

3.5.ii) Let us consider case $(3.39)$ and $\lambda \neq 0$. We substitute $r_{n}$ into system (3.59)-(3.61) and into equation (3.37), we get the following system:

$$
\begin{aligned}
& A_{1} e^{u_{0}-h u_{-1}}+B_{1} e^{u_{1}-h u_{0}}=0, \\
& A_{2} e^{u_{0}-h u_{-1}}+B_{2} e^{u_{1}-h u_{0}}=0, \\
& A_{3} e^{u_{0}-h u_{-1}}+B_{3} e^{u_{1}-h u_{0}}=0, \\
& A_{4} e^{u_{1}-h u_{0}}+B_{4} e^{u_{2}-h u_{1}}=0 .
\end{aligned}
$$

Obviously, the coefficients $A_{i}, B_{i}$ at independent exponent functions have to be equal to zero. Thus, we obtain a system of 8 algebraic equations in 8 unknowns $c, h, M, \lambda, \rho, \kappa, k, \nu_{k}$. Studying this system, we get the following possible variants:

$$
M=4, \quad k=\frac{10}{3} ; \quad M=5, \quad k=\frac{17}{4} ; \quad M=2, \quad k=2 .
$$

All of these variants contradict our assumptions about values of $k, M$.

Thus, we have proved the following statement.

Lemma 3.7. If relations (3.9), (3.34), 3.45) hold true for some $M \geqslant 2, k \geqslant 2, N \geqslant 2$, then the function $r_{n}$ casts into one of the forms (3.50) or (3.68) up to point transformations.

Lemma 2.2 is implied immediately by Lemma 3.7.

Summarizing the rezults of this section, we observe that we have lattice (1.2) for further study, where ther function $r_{n}$ is defined by one of the formulae $r_{n}=r_{n}\left(u_{n}\right),(3.50),(3.68)$. Similarly, function $p_{n}$ is defined by one of the following formulae:

$$
\begin{aligned}
& p_{n}=p_{n}\left(u_{n}\right), \\
& p_{n}\left(u_{n+1}, u_{n}, u_{n-1}\right)=e^{u_{n}-u_{n-1}}-e^{u_{n+1}-u_{n}}, \\
& p_{n}\left(u_{n+1}, u_{n}, u_{n-1}\right)=-u_{n+1}+2 u_{n}-u_{n-1} .
\end{aligned}
$$

\section{FUNCTION $q_{n}$}

We recall that the operator $Y$ can be represented as follows, see formula (2.3):

$$
Y=\sum_{i} u_{i, y} Y_{i}+R
$$

where

$$
\begin{aligned}
Y_{i} & =\frac{\partial}{\partial u_{i}}+r_{i} \frac{\partial}{\partial u_{i, x}}+\left(D_{x}\left(r_{i}\right)+r_{i}^{2}\right) \frac{\partial}{\partial u_{i, x x}}+\cdots \\
R & =\sum_{i}\left(u_{i, x} p_{i}+q_{i}\right) \frac{\partial}{\partial u_{i, x}}+\left(D_{x}\left(u_{i, x} p_{i}+q_{i}\right)+\left(u_{i, x} p_{i}+q_{i}\right) r_{i}\right) \frac{\partial}{\partial u_{i, x x}}+\cdots
\end{aligned}
$$

We shall determine the function $q_{n}$ by using the operator $R$. We define a sequence of operators in the characteristic algebra $\mathcal{L}(y, N)$ by the following recurrent formula:

$$
\begin{gathered}
Y_{-1}, \quad Y_{0}, \quad Y_{1}, \quad Y_{0,-1}=\left[Y_{0}, Y_{-1}\right], \quad Y_{1,0}=\left[Y_{1}, Y_{0}\right] \\
R_{0}=\left[Y_{0}, R\right], \quad R_{1}=\left[Y_{0}, R_{0}\right], \quad R_{2}=\left[Y_{0}, R_{1}\right], \quad \ldots \quad R_{k+1}=\left[Y_{0}, R_{k}\right] .
\end{gathered}
$$


For elements of the sequence the following commutation relations hold:

$$
\begin{aligned}
& {\left[D_{x}, Y_{-1}\right]=-r_{-1} Y_{-1}, \quad\left[D_{x}, Y_{0}\right]=-r_{0} Y_{0}, \quad\left[D_{x}, Y_{1}\right]=-r_{1} Y_{1},} \\
& {\left[D_{x}, Y_{0,-1}\right]=-\left(r_{-1}+r_{0}\right) Y_{0,-1}-Y_{0}\left(r_{-1}\right) Y_{-1}+Y_{-1}\left(r_{0}\right) Y_{0},} \\
& {\left[D_{x}, Y_{1,0}\right]=-\left(r_{0}+r_{1}\right) Y_{1,0}-Y_{1}\left(r_{0}\right) Y_{0}+Y_{0}\left(r_{1}\right) Y_{1},} \\
& {\left[D_{x}, R\right]=-\sum_{i} h_{i} Y_{i}, \quad h_{i}=p_{i} u_{i, x}+q_{i},} \\
& {\left[D_{x}, R_{0}\right]=-r_{0} R_{0}+h_{1} Y_{1,0}-h_{-1} Y_{0,-1}} \\
& \quad-Y_{0}\left(h_{1}\right) Y_{1}+\left(R\left(r_{0}\right)-Y_{0}\left(h_{0}\right)\right) Y_{0}-Y_{0}\left(h_{-1}\right) Y_{-1}, \\
& {\left[D_{x}, R_{1}\right]=-2 r_{0} R_{1}-Y_{0}\left(r_{0}\right) R_{0}+\cdots} \\
& {\left[D_{x}, R_{2}\right]=-3 r_{0} R_{2}-3 Y_{0}\left(r_{0}\right) R_{1}-Y_{0}^{2}\left(r_{0}\right) R_{0}+\cdots,} \\
& {\left[D_{x}, R_{3}\right]=-4 r_{0} R_{3}-6 Y_{0}\left(r_{0}\right) R_{2}-4 Y_{0}^{2}\left(r_{0}\right) R_{1}-Y_{0}^{3}\left(r_{0}\right) R_{0}+\cdots,}
\end{aligned}
$$

where the dots stand for a linear combinations of operators $Y_{1,0}, Y_{0,-1}, Y_{1}, y_{0}, Y_{-1}$. By induction we prove that the following formula holds for all $n \geqslant 2$ :

$$
\left[D_{x}, R_{n}\right]=a_{n} R_{n}+b_{n} R_{n-1}+\cdots,
$$

where

$$
a_{n}=-(n+1) r_{0}, \quad b_{n}=-\frac{n^{2}+n}{2} Y_{0}\left(r_{0}\right),
$$

and the dots stand for a linear combinations of the operators $R_{k}, k<n-1, Y_{1,0}, Y_{0,-1}, Y_{1}, Y_{0}, Y_{-1}$.

Lemma 4.1. If the operator $R_{0}$ is linearly expressed in terms of operators 4.1.

$$
R_{0}=\mu Y_{1,0}+\tilde{\mu} Y_{0,-1}+\nu Y_{1}+\eta Y_{0}+\varepsilon Y_{-1},
$$

then chain (1.2) is reduced to one of forms (2.7), (2.8) by point transformations.

Proof. We apply the operator $\operatorname{ad}_{D_{x}}$ to both sides of identity (4.2). Collecting the coefficients at independent operators $Y_{1,0}, Y_{0,-1}, Y_{1}, Y_{0}, Y_{-1}$, we get the system of equations

$$
\begin{aligned}
& D_{x}(\mu)=r_{1} \mu+h_{1}, \\
& D_{x}(\tilde{\mu})=r_{-1} \tilde{\mu}-h_{-1}, \\
& D_{x}(\nu)=\left(r_{1}-r_{0}\right) \nu-Y_{0}\left(h_{1}\right)-\mu Y_{0}\left(r_{1}\right), \\
& D_{x}(\eta)=R\left(r_{0}\right)-Y_{0}\left(h_{0}\right)+\mu Y_{1}\left(r_{0}\right)-\tilde{\mu} Y_{-1}\left(r_{0}\right), \\
& D_{x}(\varepsilon)=\left(r_{-1}-r_{0}\right) \varepsilon-Y_{0}\left(h_{-1}\right)+\tilde{\mu} Y_{0}\left(r_{-1}\right) .
\end{aligned}
$$

We consider equation 4.3 ):

$$
r_{1}\left(u_{2}, u_{1}, u_{0}\right) \mu+p_{1}\left(u_{2}, u_{1}, u_{0}\right) u_{1, x}+q_{1}\left(u_{2}, u_{1}, u_{0}\right)=D_{x}(\mu) .
$$

A simple analysis of this equation shows that $\mu=\mu\left(u_{1}\right)$ and, hence, this equation splits into two equations

$$
\mu^{\prime}\left(u_{1}\right)=p_{1}\left(u_{2}, u_{1}, u_{0}\right), \quad r_{1}\left(u_{2}, u_{1}, u_{0}\right) \mu\left(u_{1}\right)+q_{1}\left(u_{2}, u_{1}, u_{0}\right)=0 .
$$

Hence,

$$
p_{n}\left(u_{n+1}, u_{n}, u_{n-1}\right)=\mu^{\prime}\left(u_{n}\right), \quad q_{n}\left(u_{n+1}, u_{n}, u_{n-1}\right)=-r_{n}\left(u_{n+1}, u_{n}, u_{n-1}\right) \mu\left(u_{n}\right) .
$$

Using equation (4.4), we obtain that $\tilde{\mu}=\tilde{\mu}\left(u_{-1}\right), \tilde{\mu}(v)=-\mu(v)$.

We simplify identity 4.5 using 4.8 and we get

$$
D_{x}(\nu)=\left(r_{1}-r_{0}\right) \nu .
$$

It easy to see that $\nu=0$. Similarly, it follows from (4.7) that $\varepsilon=0$.

We simplify identity 4.6 as follows:

$$
D_{x}(\eta)=-p_{0, u_{0}} u_{0, x}-q_{0, u_{0}}-r_{0} p_{0}+\mu r_{0, u_{1}}-\tilde{\mu} r_{0, u_{-1}} .
$$


A simple analysis of this equation shows that $\eta=\eta\left(u_{0}\right)$ and, hence, this equation splits into two equations

$$
\eta^{\prime}\left(u_{0}\right)=-p_{0, u_{0}}, \quad-q_{0, u_{0}}-r_{0} p_{0}+\mu r_{0, u_{1}}-\tilde{\mu} r_{0, u_{-1}}=0 .
$$

We substitute formulae (4.8) into identities (4.9) and we obtain $\eta^{\prime}\left(u_{0}\right)=-\mu^{\prime \prime}\left(u_{0}\right)$,

$$
r_{0, u_{0}} \mu\left(u_{0}\right)+\mu\left(u_{1}\right) r_{0, u_{1}}+\mu\left(u_{-1}\right) r_{0, u_{-1}}=0 .
$$

We substitute the function $r_{n}$ defined by formula (3.50) into (4.10), and we get that $\mu=c$ is an arbitrary constant. Therefore, $p_{n}=0, q_{n}=-c r_{n}$, and lattice (1.2) becomes

$$
u_{n, x y}=\left(e^{u_{n}-u_{n-1}}-e^{u_{n+1}-u_{n}}\right) u_{n, y}-c\left(e^{u_{n}-u_{n-1}}-e^{u_{n+1}-u_{n}}\right) .
$$

The transformation $u_{n}-c y \rightarrow u_{n}$ reduces this lattice to (2.7).

If $r_{n}$ is defined by (3.68), then (4.10) implies $\mu=c$, where $c$ is an arbitrary constant. Hence, $p_{n}=0, q_{n}=-c r_{n}$, and lattice (1.2) takes the following form:

$$
u_{n, x y}=\left(-u_{n+1}+2 u_{n}-u_{n-1}\right) u_{n, y}+c\left(-u_{n+1}+2 u_{n}-u_{n-1}\right) \text {. }
$$

The transformation $u_{n}-c y \rightarrow u_{n}$ reduces this lattice to (2.8).

If $r_{n}=r_{n}\left(u_{n}\right)$, then it follows from 4.10 that $\mu=0$ or $r_{0, u_{0}}=0$. In the first case formulae (4.8) imply $p_{n}=0, q_{n}=0$. Then chain (1.2) becomes $u_{n, x y}=r_{n}\left(u_{n}\right) u_{n, y}$. In the second case $r_{0}=c_{1}$, where $c_{1}$ is an arbitrary constant, hence, by 4.80$), p_{n}=\mu^{\prime}\left(u_{n}\right), q_{n}=-c_{1} \mu\left(u_{n}\right)$, and chain (1.2) casts into the form $u_{n, x y}=\mu^{\prime}\left(u_{n}\right) u_{n, x}+c_{1} u_{n, y}-c_{1} \mu\left(u_{n}\right)$. The proof is complete.

Suppose that $R_{n}$ depends linearly on $R_{k}, k<n, Y_{1,0}, Y_{0,-1}, Y_{1}, Y_{0}, Y_{-1}$ for some $n$ :

$$
R_{n}=\lambda R_{n-1}+\cdots, \quad n>0 .
$$

Lemma 4.2. If function $r_{n}$ has one of forms (3.50, (3.68), then case 4.11) is not realized. Proof. We apply the operator $\operatorname{ad}_{D_{x}}$ to both sides of identity 4.11). Collecting coefficients at $R_{n-1}$ in obtained relation, we get the equation:

$$
D_{x}(\lambda)=-r_{0} \lambda-\frac{n^{2}+n}{2} r_{0, u_{0}} .
$$

A simple analysis of this equation shows that $\lambda$ is a constant, hence

$$
r_{0} \lambda+\frac{n^{2}+n}{2} r_{0, u_{0}}=0
$$

Substituting formulae (3.50), 3.68) into (4.12), we get that $\lambda=0$ and $n^{2}+n=0$. Hence, $n=0$ or $n=-1$. Both solutions contradict the assumption $n>0$. The proof is complete.

heorem 1.1 is implied Lemma 4.1 , 4.2 .

\section{Appendix. Proof of Lemma 3.6}

The proof is a study of equation (3.37):

$$
\nu_{k} r_{1}+\frac{k(k-1)}{2} r_{1, u_{1}}+(k-1)\left(r_{0, u_{1}}+r_{2, u_{1}}\right)=0
$$

in different cases (3.10) and (3.11) under conditions (1.4), $M \geqslant 2, k \geqslant 2$. We denote $\nu_{k}=\nu$.

i) We substitute function $r_{0}$ defined by formula (3.10) into (5.1)

$$
\nu\left(\alpha\left(u_{0}\right)-\frac{2}{M-1} \alpha\left(u_{1}\right)+\delta\left(u_{2}\right)\right)-\frac{k(k-1)}{M-1} \alpha^{\prime}\left(u_{1}\right)+(k-1)\left(\delta^{\prime}\left(u_{1}\right)+\alpha^{\prime}\left(u_{1}\right)\right)=0 .
$$

We apply the operator $\frac{\partial}{\partial u_{2}}$ to this identity, and we get $\nu \delta^{\prime}\left(u_{2}\right)=0$. It is easy to show that the case $\nu \neq 0$ leads us to a contradiction to (1.4). Assume that $\nu=0$, then from (5.2) we obtain that the function $r_{0}$ becomes

$$
r_{0}\left(u_{1}, u_{0}, u_{-1}\right)=\alpha\left(u_{-1}\right)-\frac{2}{M-1} \alpha\left(u_{0}\right)+\left(\frac{k}{M-1}-1\right) \alpha\left(u_{1}\right)+c_{1} .
$$


ii) We substitute the function $r_{0}$ defined by formula (3.11) into equation (5.1).

$$
\begin{aligned}
& \frac{\beta\left(u_{0}\right)\left(\nu M^{2}-\nu M+k \lambda-k^{2} \lambda\right)}{M(M-1)} e^{-\frac{2 \lambda u_{1}}{M(M-1)}}+(k-1) \beta^{\prime}\left(u_{1}\right) e^{-\frac{2 \lambda u_{2}}{M(M-1)}} \\
& +\nu \psi\left(u_{1}, u_{2}\right)+(k-1) \frac{\partial \psi\left(u_{0}, u_{1}\right)}{\partial u_{1}}+\frac{1}{2}(k-1) k \frac{\partial \psi\left(u_{1}, u_{2}\right)}{\partial u_{1}}=0 .
\end{aligned}
$$

We apply the operator $\frac{\partial}{\partial u_{0}}$ to both sides of identity 5.3 .

$$
\frac{\beta^{\prime}\left(u_{0}\right)\left(\nu M^{2}-\nu M+k \lambda-k^{2} \lambda\right)}{M(M-1)} e^{-\frac{2 \lambda u_{1}}{M(M-1)}}+(k-1) \frac{\partial^{2} \psi\left(u_{0}, u_{1}\right)}{\partial u_{0} \partial u_{1}}=0 .
$$

This equation has the following solution::

$$
\psi\left(u_{0}, u_{1}\right)=\frac{\beta\left(u_{0}\right)\left(\nu M^{2}-\nu M+k \lambda-k^{2} \lambda\right)}{2(k-1) \lambda} e^{-\frac{2 \lambda u_{1}}{M(M-1)}}+F_{1}\left(u_{0}\right)+F_{2}\left(u_{1}\right) .
$$

We substitute function (5.4) into equation (5.3), then we differentiate an obtained identity with respect to $u_{2}$, and we multiple both sides of the obtained identity by $e^{\frac{2 \lambda u_{2}}{M(M-1)}}$ :

$$
\begin{aligned}
- & \frac{\nu\left(\nu M^{2}-\nu M-k^{2} \lambda+k \lambda\right) \beta\left(u_{1}\right)}{M(k-1)(M-1)} \\
& -\frac{1}{2} \frac{\left(\lambda k^{3}+k^{2} \lambda+k M^{2} \nu-k M \nu+4 k \lambda-4 \lambda\right) \beta^{\prime}\left(u_{1}\right)}{(M-1) M}+F_{2}^{\prime}\left(u_{2}\right) e^{\frac{2 \lambda u_{2}}{M(M-1)}} \nu=0 .
\end{aligned}
$$

Let us consider two different cases $\nu=0$ and $\nu \neq 0$.

ii.1) If $\nu=0$, then (5.5) becomes

$$
\frac{1}{2} \frac{\lambda(k-1)(k-2)(k+2) \beta^{\prime}\left(u_{1}\right)}{M(M-1)}=0
$$

It follows from this identity that $k=2$ or $\beta^{\prime}\left(u_{1}\right)=0$.

ii.1.1) If $k=2$, then equation (5.3) casts into the form $F_{1}^{\prime}\left(u_{1}\right)+F_{2}^{\prime}\left(u_{1}\right)=0$. It is clear that $F_{2}\left(u_{1}\right)=-F_{1}\left(u_{1}\right)+c_{1}$. Equation (3.12) becomes

$$
\begin{aligned}
& \left(-\lambda \beta\left(u_{0}\right)-\frac{1}{2} \beta^{\prime}\left(u_{0}\right) M^{2}+\frac{3}{2} M \beta^{\prime}\left(u_{0}\right)\right) e^{-\frac{2 \lambda u_{1}}{M(M-1)}} \\
& +\frac{1}{2} M(M-1) F_{1}^{\prime}\left(u_{0}\right)+\lambda\left(F_{1}\left(u_{0}\right)-F_{1}\left(u_{1}\right)+c_{1}\right)=0 .
\end{aligned}
$$

We apply the operator $\frac{\partial^{2}}{\partial u_{1} \partial u_{0}}$ to both sides of identity (5.7)

$$
\lambda e^{-\frac{2 \lambda u_{1}}{M(M-1)}} \frac{2 \lambda \beta^{\prime}\left(u_{0}\right)+M(M-3) \beta^{\prime \prime}\left(u_{0}\right)}{M(M-1)}=0 .
$$

By the condition $\lambda \neq 0$ we see that

$$
2 \lambda \beta^{\prime}\left(u_{0}\right)+M(M-3) \beta^{\prime \prime}\left(u_{0}\right)=0 .
$$

ii.1.1.1) If $M=3$, then $\beta\left(u_{0}\right)=c_{0}$, where $c_{0}$ is an arbitrary constant. The function $r_{0}$ defined by formula (3.11) becomes

$$
r_{0}\left(u_{1}, u_{0}, u_{-1}\right)=c_{0} e^{-\frac{1}{3} \lambda u_{0}}-c_{0} e^{-\frac{1}{3} \lambda u_{1}}+F_{1}\left(u_{0}\right)-F_{1}\left(u_{1}\right)+c_{1},
$$

and equation $(3.12)$ reads as

$$
-\lambda c_{0} e^{-\frac{1}{3} \lambda u_{1}}-\lambda F_{1}\left(u_{1}\right)+\lambda F_{1}\left(u_{0}\right)+\lambda c_{1}+3 F_{1}^{\prime}\left(u_{0}\right)=0 .
$$

We apply the operator $\frac{\partial}{\partial u_{1}}$ to identity 5.10 :

$$
\frac{1}{3} \lambda^{2} c_{0} e^{-\frac{1}{3} \lambda u_{1}}-\lambda F_{1}^{\prime}\left(u_{1}\right)=0,
$$


hence,

$$
F_{1}\left(u_{1}\right)=-c_{0} e^{-\frac{1}{3} \lambda u_{1}}+C_{1} .
$$

We substitute $F_{1}$ into $(5.9)$ and we get $r_{0}=C_{1}$. This contradicts condition (1.4).

ii.1.1.2) If $M \neq 3$, then equation (5.8) has the solution

$$
\beta\left(u_{0}\right)=C_{1}+C_{2} e^{-\frac{2 \lambda u_{0}}{M(M-3)}} .
$$

We differentiate equation 3.12 with respect to $u_{1}$ and, since $\lambda \neq 0$, this equation gives

$$
F_{1}\left(u_{1}\right)=-C_{1} e^{-\frac{2 \lambda u_{1}}{M(M-1)}}+C_{2} .
$$

Equation 3.12 becomes $\lambda c_{1}=0$, hence, $c_{1}=0$, and, finally,

$$
r_{0}\left(u_{1}, u_{0}, u_{-1}\right)=C_{2} e^{-\frac{2 \lambda}{M(M-1)} u_{0}-\frac{2 \lambda}{M(M-3)} u_{-1}}-C_{2} e^{-\frac{2 \lambda}{M(M-3)} u_{0}-\frac{2 \lambda}{M(M-1)} u_{1}} .
$$

We return back to equation (5.6) and consider the following case.

ii.1.2) If $\beta^{\prime}\left(u_{1}\right)=0$, then $\beta\left(u_{1}\right)=c_{3}$, where $c_{3}$ is an arbitrary constant. By equation (5.1) we find

Equation 3.12 is transformed as

$$
F_{2}\left(u_{1}\right)=-\frac{1}{2} k F_{1}\left(u_{1}\right)+c_{4} .
$$

$$
\lambda F_{1}\left(u_{0}\right)+\frac{1}{2} M(M-1) F_{1}^{\prime}\left(u_{0}\right)-\frac{1}{2} \lambda\left(c_{3} k e^{-\frac{2 \lambda u_{1}}{M(M-1)}}+k F_{1}\left(u_{1}\right)-2 c_{4}\right)=0 .
$$

We apply the operator $\frac{\partial}{\partial u_{1}}$ to both sides of identity 5.12

$$
-\frac{1}{2} \frac{k \lambda}{M(M-1)}\left(-2 c_{3} \lambda e^{-\frac{2 \lambda u_{1}}{M(M-1)}}+M(M-1) F_{1}^{\prime}\left(u_{1}\right)\right)=0 .
$$

This equation has the solution

$$
F_{1}\left(u_{1}\right)=-c_{3} e^{-\frac{2 \lambda u_{1}}{M(M-1)}}+c_{5}
$$

We substitute $F_{1}$ into (5.12), and we find $c_{4}: c_{4}=\frac{1}{2} c_{5}(k-2)$. We substitute the found functions and constants into (3.11) and we get $r_{0}\left(u_{1}, u_{0}, u_{-1}\right)=0$, which contradicts condition (1.4).

We return back to equation (5.5).

ii.2) If $\nu \neq 0$, then $F_{2}^{\prime}\left(u_{2}\right) e^{\frac{2 \lambda u_{2}}{M(M-1)}}=c_{1}$ and, hence,

$$
F_{2}\left(u_{2}\right)=-\frac{1}{2} \frac{M(M-1) c_{1} e^{-\frac{2 \lambda u_{2}}{M(M-1)}}}{\lambda}+c_{2} .
$$

Equation (5.5) reads as

$$
\begin{aligned}
- & \frac{\nu\left(\nu M^{2}-\nu M-k^{2} \lambda+k \lambda\right) \beta\left(u_{1}\right)}{M(k-1)(M-1)} \\
-\frac{1}{2} \frac{\left(\lambda k^{3}+k^{2} \lambda+k M^{2} \nu-k M \nu+4 k \lambda-4 \lambda\right) \beta^{\prime}\left(u_{1}\right)}{(M-1) M}+c_{1} \nu & =0 .
\end{aligned}
$$

We denote:

$$
\begin{aligned}
& A=\nu M^{2}-\nu M-k^{2} \lambda+k \lambda, \\
& B=\lambda k^{3}+k^{2} \lambda+k M^{2} \nu-k M \nu+4 k \lambda-4 \lambda .
\end{aligned}
$$

We shall consider the following different cases:
ii.2.1) $A=0, B=0$;
ii.2.2) $A=0, B \neq 0$;
ii.2.3) $A \neq 0, B=0$
ii.2.4) $A \neq 0, B \neq 0$. 
In case ii.2.1), that is, as

$$
\nu M^{2}-\nu M-k^{2} \lambda+k \lambda=0, \quad \lambda k^{3}+k^{2} \lambda+k M^{2} \nu-k M \nu+4 k \lambda-4 \lambda=0 .
$$

Then we express $\nu$ from the first equation and we substitute this function into the second equation, and we get $4(k-1) \lambda=0$, which contradicts to $k \geqslant 2, \lambda \neq 0$.

ii.2.2) Assume that

$$
A=\nu M^{2}-\nu M-k^{2} \lambda+k \lambda=0 .
$$

We express $\nu$ from this identity and we substitute $\nu$ into (5.13). This equation has the solution $\beta\left(u_{1}\right)=\frac{1}{2} k c_{1} u_{1}+c_{3}$. Equation (5.3) becomes

$$
\frac{1}{2} k(k-1) \frac{d F_{1}\left(u_{1}\right)}{d u_{1}}+\frac{k(k-1) \lambda}{M(M-1)} F_{1}\left(u_{1}\right)+c_{1}(k-1) e^{-\frac{2 \lambda u_{1}}{M(M-1)}}+\frac{k(k-1) c_{2} \lambda}{M(M-1)}=0 .
$$

This equation has the solution

$$
F_{1}\left(u_{1}\right)=-\frac{\left(2 c_{1} u_{1}-c_{4} k\right)}{k} e^{-\frac{2 \lambda u_{1}}{M(M-1)}}-c_{2} .
$$

Equation 3.12 casts into the form

$$
-\frac{c_{1} M(M-1)}{k} e^{-\frac{2 \lambda u_{0}}{M(M-1)}}-\frac{c_{1} M(M-k-1)}{2} e^{-\frac{2 \lambda u_{1}}{M(M-1)}}=0 .
$$

It is clear that this identity holds true only if $c_{1}=0$ (we are working under the condition $M \geqslant 2)$. Hence, we have

$$
r_{0}\left(u_{1}, u_{0}, u_{-1}\right)=\left(c_{3}+c_{4}\right) e^{-\frac{2 \lambda u_{0}}{M(M-1)}},
$$

which contradicts condition (1.4).

ii.2.3) Suppose that

$$
B=\lambda k^{3}+k^{2} \lambda+k M^{2} \nu-k M \nu+4 k \lambda-4 \lambda=0 .
$$

We express $\nu$ :

$$
\nu=\frac{\lambda(k-1)(k-2)(k+2)}{k M(M-1)} .
$$

Since $\nu \neq 0$, then $k \neq 2$. Equation $(5.13)$ becomes

$$
\frac{\lambda(k-1)(k-2)(k+2)}{k^{2} M^{2}(M-1)^{2}}\left(c_{1} k M^{2}-c_{1} k M+4 \lambda \beta\left(u_{1}\right)\right)=0 .
$$

We find the function $\beta$ :

$$
\beta\left(u_{1}\right)=-\frac{1}{4} \frac{c_{1} k M(M-1)}{\lambda} .
$$

Taking into consideration the obtained function, we simplify equation (5.3):

$$
\begin{aligned}
\frac{1}{2} k(k-1) \frac{d F_{1}\left(u_{1}\right)}{d u_{1}} & +\frac{\lambda(k-1)(k-2)(k+2)}{k M(M-1)} F_{1}\left(u_{1}\right) \\
& +(k-1) c_{1} e^{-\frac{2 \lambda u_{1}}{M(M-1)}}+\frac{c_{2} \lambda(k-2)(k-1)(k+2)}{k M(M-1)}=0 .
\end{aligned}
$$

This equation has the solution:

$$
F_{1}\left(u_{1}\right)=\frac{c_{1} k M(M-1)}{4 \lambda} e^{-\frac{2 \lambda u_{1}}{M(M-1)}}+C_{1} e^{-\frac{2 \lambda(k-2)(k+2)}{k^{2} M(M-1)} u_{1}}-c_{2} .
$$

Let us transform equation 3.12

$$
\frac{4 \lambda C_{1}}{k^{2}} e^{-\frac{2 \lambda(k-2)(k+2)}{k^{2} M(M-1)} u_{1}}=0 .
$$

It follows from this identity that $C_{1}=0$. Substitution found functions and constants into (3.11), we obtain that $r_{0}=0$, that contradicts condition (1.4). 
ii.2.4) If $A \neq 0$ and $B \neq 0$, then equation (5.13) has the solution

$$
\beta\left(u_{1}\right)=c_{3} e^{-\frac{2 \nu\left(\nu M^{2}-\nu M-k^{2} \lambda+k \lambda\right) u_{1}}{(k-1)\left(-\lambda k^{3}+k^{2} \lambda+4 k \lambda+k M^{2} \nu-k M \nu-4 \lambda\right)}}+\frac{M c_{1}(k-1)(M-1)}{\nu M^{2}-\nu M-k^{2} \lambda+k \lambda} .
$$

We substitute (5.17) into (5.3) and we obtain

$$
\frac{1}{2} k(k-1) \frac{d F_{1}\left(u_{1}\right)}{d u_{1}}+\nu F_{1}\left(u_{1}\right)+c_{1}(k-1) e^{-\frac{2 \lambda u_{1}}{M(M-1)}}+\nu c_{2} .
$$

Equation (5.18) has the solution

$$
F_{1}\left(u_{1}\right)=-c_{2}+c_{4} e^{-\frac{2 \nu u_{1}}{k(k-1)}}-\frac{c_{1} M(M-1)(k-1)}{\nu M^{2}-\nu M-k^{2} \lambda+k \lambda} e^{-\frac{2 \lambda u_{1}}{M(M-1)}} .
$$

Function (3.11) becomes

$$
r_{0}\left(u_{1}, u_{0}, u_{-1}\right)=c_{4} e^{-\frac{2 \nu u_{0}}{k(k-1)}}+c_{3} e^{-\frac{2 \lambda u_{0}}{M(M-1)}-\frac{2 \nu A u_{-1}}{B(k-1)}}+\frac{A c_{3}}{2(k-1) \lambda} e^{-\frac{2 \lambda u_{1}}{M(M-1)}-\frac{2 \nu A u_{0}}{B(k-1)}} .
$$

Here $A, B$ are defined by formulae (5.14), (5.15). We substitute these functions into (3.12)

$$
-\frac{A c_{4}}{k(k-1)} e^{-\frac{2 \nu u_{0}}{k(k-1)}}-\frac{A c_{3}\left(\lambda B+\nu M^{2} A-\nu M A-\lambda k B-4 M \nu \lambda+4 M \nu k \lambda\right)}{2 B k \lambda(k-1)^{2}} e^{-\frac{2 \lambda u_{1}}{M(M-1)}-\frac{2 \nu A u_{0}}{B(k-1)}}=0 .
$$

Since $A \neq 0, \nu \neq 0$, it follows from the last identity that $c_{4}=0$ and

$$
\lambda B+\nu M^{2} A-\nu M A-\lambda k B-4 M \nu \lambda+4 M \nu k \lambda=0 .
$$

Thus, we have specified the function $r_{0}$ :

$$
r_{0}\left(u_{1}, u_{0}, u_{-1}\right)=c_{3} e^{-\frac{2 \lambda u_{0}}{M(M-1)}-\frac{2 \nu A u_{-1}}{B(k-1)}}+\frac{A c_{3}}{2(k-1) \lambda} e^{-\frac{2 \lambda u_{1}}{M(M-1)}-\frac{2 \nu A u_{0}}{B(k-1)}} .
$$

We can rewrite $r_{0}$ in the following form:

$$
r_{0}\left(u_{1}, u_{0}, u_{-1}\right)=C_{1} e^{h_{1} u_{0}-h_{2} u_{-1}}+C_{2} e^{h_{1} u_{1}-h_{2} u_{0}},
$$

where $C_{1} C_{2} \neq 0, h_{1} h_{2} \neq 0$ are some constants.

Lattice (1.2) is reduced to one with $r_{n}$ of the following form

$$
r_{n}\left(u_{n+1}, u_{n}, u_{n-1}\right)=e^{u_{n}-h u_{n-1}}+c e^{u_{n+1}-h u_{n}}
$$

by rescaling $h_{1} u_{n} \rightarrow u_{n}, c_{1} h_{1} x \rightarrow x$. Similarly transformations one can apply to the lattice in case ii.1.1.2 (see (5.11)). The proof of Lemma 3.6 is complete.

\section{REFERENCES}

1. E.V. Ferapontov, K.R. Khusnutdinova. On the integrability of (2+1)-dimensional quasilinear systems // Comm. Math. Phys. 248:1 (2004), 187-206.

2. E. V. Ferapontov, K. R. Khusnutdinova, M. V. Pavlov. Classification of integrable $(2+1)-$ dimensional quasilinear hierarchies // Theor. Math. Phys. 144:1 (2005), 907-915.

3. E.V. Ferapontov, K.R. Khusnutdinova, S.P. Tsarev. On a class of three-dimensional integrable Lagrangians // Comm. Math. Phys. 261:1, 225-243 (2006).

4. A.V. Odesskii, V.V. Sokolov. Integrable $(2+1)$-dimensional systems of hydrodynamic type // Theor. Math. Phys. 163:2, 549-586 (2010).

5. L.V. Bogdanov, B. G. Konopelchenko. Grassmannians $\operatorname{Gr}(N-1, N+1)$, closed differential $N-$ 1 forms and $N$-dimensional integrable systems // J. Phys. A: Math. Theor. 46:8, id 085201 (2013).

6. M.V. Pavlov,Z. Popowicz. On integrability of a special class of two-component (2+1)-dimensional hydrodynamic-type systems // SIGMA 5, id 011 (2009).

7. A.K. Pogrebkov. Commutator identities on associative algebras and the integrability of nonlinear evolution equations // Theor. Math. Phys. 154:3, 405-417 (2008).

8. M. Mañas, L.M. Alonso, C. Álvarez-Fernández. The multicomponent 2D Toda hierarchy: discrete flows and string equations // Inverse Problems 25, id 065007 (2009). 
9. V.E. Zakharov, S.V. Manakov. Construction of higher-dimensional nonlinear integrable systems and of their solutions // Funct. Anal. Appl. 19:2, 89-101 (1985).

10. I.S. Krasil'shchik, A. Sergyeyev, O.I. Morozov. Infinitely many nonlocal conservation laws for the $A B C$ equation with $A+B+C \neq 0 / /$ Calc. Var. PDEs. 55:5, id 123 (2016).

11. A.B. Shabat, R.I. Yamilov. Exponential systems of type I and the Cartan matrix Preprint, Bashkir branch of AS USSR, Ufa (1981). (in Russian).

12. A. N. Leznov, V. G. Smirnov, A. B. Shabat. The group of internal symmetries and the conditions of integrability of two-dimensional dynamical systems. // Theor. Math. Phys. 51:1, 322-330 (1982).

13. F.H. Mukminov, A.V. Zhiber. Quadratic systems, symmetries, characteristic and complete algebras // in "Problems of mathematical physics and the asymptotics of their solutions". Bashkir Scientific Center of the Ural Branch AS USSR, Ufa, 14-32 (1991). (in Russian)

14. A.V. Zhiber, R.D. Murtazina, I.T. Habibullin, A.B. Shabat, Characteristic Lie rings and nonlinear integrable equations. Inst. Comp. Stud., Moscow (2012). (in Russian)

15. A.V. Zhiber, R.D. Murtazina, I.T. Habibullin, A.B. Shabat, Characteristic Lie rings and integrable models in mathematical physics // Ufa Math. J. 4:3, 17-85 (2012).

16. I.T. Habibullin. Characteristic Lie rings, finitely-generated modules and integrability conditions for (2+ 1)-dimensional lattices // Physica Scripta. 87:6, id 065005 (2013).

17. I.T. Habibullin, M.N. Poptsova(Kuznetsova). Classification of a subclass of two-dimensional lattices via characteristic lie rings // SIGMA 13, 073 (2017).

18. I. T. Habibullin, M. N. Poptsova(Kuznetsova). Algebraic properties of quasilinear two-dimensional lattices connected with integrability // Ufa Math. J. 10:3, 86-105(2018).

19. A.B. Shabat. Higher symmetries of two-dimensional lattices // Phys. Lett. A. 200:2, 121-133 (1995).

20. G. Rinehart. Differential forms for general commutative algebras // Trans. Amer.Math. Soc. 108:2, 195-222 (1963).

21. D. Millionshchikov. Lie Algebras of Slow Growth and Klein-Gordon PDE // Algebras Represent. Theor. 21:5, 1037-1069 (2018).

22. A.B. Shabat, R.I. Yamilov. To a transformation theory of two-dimensional integrable systems // Phys. Lett. A. 227:1-2, 15-23 (1997).

23. R. Yamilov. Symmetries as integrability criteria for differential difference equations // J. Phys. A: Math. Gen. 39:45, 541-623 (2006).

24. I.T. Habibullin, A. Pekcan. Characteristic Lie algebra and classification of semidiscrete models // Theor. Math. Phys. 151:3, 781-790 (2007).

25. A.N. Leznov, V.G. Smirnov, A.B. Shabat. The group of internal symmetries and the conditions of integrability of two-dimensional dynamical systems // Theor. Math. Phys. 51:1, 322-330 (1982).

Mariya Nikolaevna Kuznetsova,

Institute of Mathematics,

Ufa Federal Research Center,

Russian Academy of Sciences,

Chernyshevsky str. 112,

450008, Ufa, Russia

E-mail: mariya.n.kuznetsova@gmail.com 